\title{
SmartPulse, a Machine Learning Approach for Calibration-Free Dynamic RF Shimming: Preliminary Study in a Clinical Environment
}

\section{Authors}

Raphaël Tomi-Tricot, PhD; ${ }^{1}$ Vincent Gras, PhD; ${ }^{1}$ Bertrand Thirion, PhD; ${ }^{2}$ Franck Mauconduit, PhD; ${ }^{3}$ Nicolas Boulant, PhD; ${ }^{1}$ Hamza Cherkaoui, MSc; ${ }^{2}$ Pierre Zerbib, BS; ${ }^{4}$ Alexandre Vignaud, PhD; ${ }^{1}$ Alain Luciani, MD, $\mathrm{PhD} ;{ }^{4,5,6}$ Alexis Amadon, $\mathrm{PhD}^{1}$

\section{Corresponding author}

Alexis Amadon, PhD

CEA, NeuroSpin, Bat. 145

Tel.: 0169087905

Gif-sur-Yvette

Fax: 0169087855

91191 Cedex

E-mail: alexis.amadon@cea.fr

France

\section{Institutions}

${ }^{1}$ NeuroSpin, CEA, Université Paris-Saclay, Bat. 145, 91191 Gif-sur-Yvette Cedex, France

2 Parietal, Inria, Université Paris-Saclay, Bat. 145, 91191 Gif-sur-Yvette Cedex, France

3 Siemens Healthcare SAS, 40 avenue des Fruitiers, 93210 Saint-Denis, France

${ }^{4}$ Department of Radiology, AP-HP, CHU Henri Mondor, 51 avenue du Maréchal de Lattre de Tassigny, 94010 Créteil Cedex, France

${ }^{5}$ Université Paris-Est Créteil Val de Marne, 61 avenue du Général de Gaulle, 94010 Créteil Cedex, France ${ }^{6}$ INSERM U955, Team 18, Molecular Virology and Immunology Physiopathology and Therapeutic of Chronic Viral Hepatitis, 8 rue du Général Sarrail, 94010 Créteil Cedex, France 


\section{Abstract}

Purpose: A calibration-free pulse design method is introduced to alleviate $\mathrm{B}_{1}{ }^{+}$ artifacts in clinical routine with parallel transmission at high field, dealing with significant inter-subject variability, found for instance in the abdomen.

Theory and Methods: From a dual-transmit 3T scanner, a database of $\mathrm{B}_{1}{ }^{+}$and offresonance abdominal maps from 50 subjects was first divided into three clusters based on mutual affinity between their respective tailored $\mathrm{k}_{\mathrm{T}}$-points pulses. For each cluster, a kT-points pulse was computed, minimizing normalized root-mean-square flip angle (FA) deviations simultaneously for all subjects comprised in it. Using 30 additional subjects' field distributions, a machine learning classifier was trained on this 80-labelled-subject database to recognize the best pulse from the three ones available, relying only on patient features accessible from the preliminary localizer sequence present in all protocols. This so-called SmartPulse process was experimentally tested on an additional 53-subject set and compared with other pulse types: vendor's hard calibration-free dual excitation, tailored static RF shimming, universal and tailored $\mathrm{kT}_{\mathrm{T}}$ points pulses.

Results: SmartPulse outperformed both calibration-free approaches. Tailored static RF shimming yielded similar FA homogeneity for most patients but broke down for some while SmartPulse remained robust. Although FA homogeneity was systematically better with tailored $\mathrm{kT}$-points, the difference was barely noticeable on in-vivo images.

Conclusion: The proposed method paves the way towards an efficient trade-off between tailored and universal pulse design approaches for large inter-subject variability. With no need for on-line field mapping or pulse design, it can fit seamlessly into a clinical protocol.

Keywords: $\mathrm{B}_{1}{ }^{+}$inhomogeneity; $\mathrm{kT}$-points; universal pulses; machine learning; abdomen; $3 \mathrm{~T}$ 


\section{INTRODUCTION}

High field magnetic resonance imaging (MRI) has proved its utility in clinical routine thanks to the high signal-to-noise ratio (SNR) it provides, allowing finer temporal and/or spatial resolutions (e.g. (1)). However, a number of problems inherent to high field still hamper the spread of $3 \mathrm{~T}$ scanners in routine everyday practice. Among them is the so-called " $\mathrm{B}_{1}{ }^{+}$artifact" that occurs when the RF wavelength gets close to, or smaller than, the imaged region (2-4). In such a case, zones of shade and loss of contrast appear, which can affect diagnosis by hiding pathologies or by altering the observed enhancement ratio in contrast-agent-injected sequences. At $3 \mathrm{~T}$, this artifact is particularly noticeable in abdominal imaging.

Both passive (5-7) and active RF shimming methods have been implemented to counteract $\mathrm{B}_{1}{ }^{+}$artifacts, at high $(4,8-10)$ and ultra-high (11-16) field (UHF: $7 T$ and above) for various imaging regions and contrast weightings. Active RF shimming makes use of parallel transmission $(\mathrm{pTx})(17,18)$, which is widely used on research scanners at UHF with eight transmission channels or more, and has been made available on some clinical $3 T$ scanners, with two channels. In that case, only static RF shimming (3) is currently proposed commercially: the user can choose between a "standard" shimming with fixed RF complex weights $(8,19)$, and a "tailored' one exploiting the measurement of the $\mathrm{B}_{1}{ }^{+}$fields from each transmit channel to adapt the coefficients to the patient in the magnet. In a previous study (10), the superiority of kT-points (14) dynamic RF shimming (3) was demonstrated in clinical routine for nonselective excitation of the abdomen at $3 \mathrm{~T}$. Indeed, although tailored static RF shimming is satisfactory for most patients, it fails to offer sufficiently homogeneous excitation in about 10 to $20 \%$ of the population. However, both techniques are tailored, i.e. they require calibration consisting at least in the measurement of $\mathrm{B}_{1}{ }^{+}$maps from each transmit channel; for optimal dynamic RF shimming, a frequency off-resonance $\Delta f_{0}$ map is also required before computation of the RF pulse. All these maps are acquired at the beginning of the examination and thus may become inaccurate in case of subject motion or/and breathing.

On a 3T scanner with two channels, the whole calibration process can last nearly two minutes: 30 seconds for $\mathrm{B}_{1}{ }^{+}$mapping, 15 seconds for $\Delta \mathrm{f}_{0}$ mapping, and between 5 seconds (static RF shimming) and 60 seconds ( $\mathrm{k}$-points) for pulse design itself. This time naturally scales with the number of transmit channels $(20,21)$. For this 
reason, Gras et al. (22) introduced the concept of universal pulses, that allows calibration-free pTx: instead of designing a pulse specific to each subject, a pulse robust to inter-subject variability is created once and for all using calibration data of a population of subjects. Universal pulses were successfully implemented in the brain at $7 \mathrm{~T}$, with a variety of sequences and weightings, and different underlying pulse designs - kT-points (22-24), fast-kz spokes (23), direct signal control (25) - and at $3 \mathrm{~T}$ in the brain (26).

Considering that universality could compromise individual homogeneity, some authors have explored machine learning approaches to design tailored pulses while reducing calibration time. lanni et al. for instance developed a method to infer RF coefficients of a static RF shimming pulse using geometrical features of the head and limited $\mathrm{B}_{1}{ }^{+}$information (27) with good accuracy. Mirfin et al. investigated a neural network solution to predict dynamic RF shimming pulses (28), but the produced pulses still lacked performance. Both methods were targeting slice-specific pulses for brain examinations at $7 \mathrm{~T}$.

The present work constitutes a proof-of-concept for a new method, SmartPulse, proposed to achieve the simplicity and user-friendliness of universal pulses under the important inter-subject variability found, for instance, in the abdomen at $3 \mathrm{~T}$ as shown in Figure 1, where a universal design may not suffice. Instead of designing one pulse for all subjects, a population is divided into clusters, and one smart-universal pulse is designed for each cluster. Then a machine learning algorithm classifies new subjects to assign the best possible pulse to each one of them. To comply with the calibration-free philosophy of universal pulses, only features that can be extracted automatically from a localizer acquisition - a compulsory step preceding all imaging protocols - are used: no additional data is needed.

In continuation of our work (10), this study was led in the context of Dynamic Contrast-Enhanced (DCE) MRI, an essential part of abdominal imaging protocols (29), consisting of a succession of 3D $T_{1}$-weighted fat-suppressed FLASH sequences before and at several points in time after gadolinium-based contrast agent injection, to follow the perfusion of tissues and characterize certain lesions $(30,31)$. КT-points being nonselective, they are appropriate for this sequence where short echo time and repetition time are required: they were therefore chosen as the underlying pulse parametrization for SmartPulse. The performance of this method was assessed on 
calibration data from various subjects and compared to several other pulses, including a universal one. The effect on DCE images acquired on a $3 \mathrm{~T}$ scanner in clinical routine was also investigated.

a. channel 1

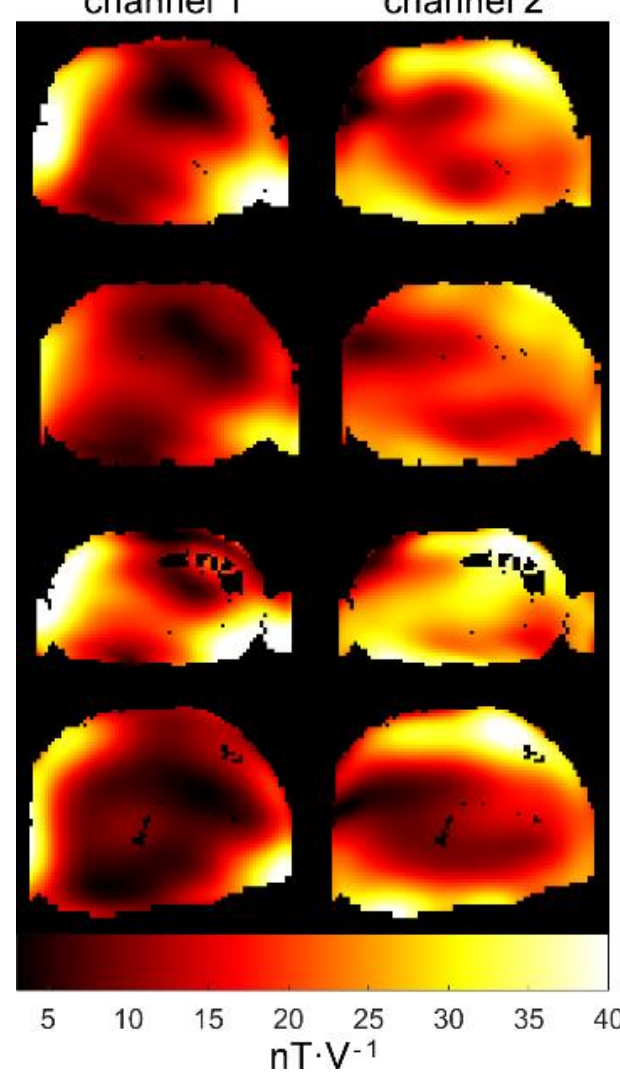

b. channel $1 \quad$ channel 2

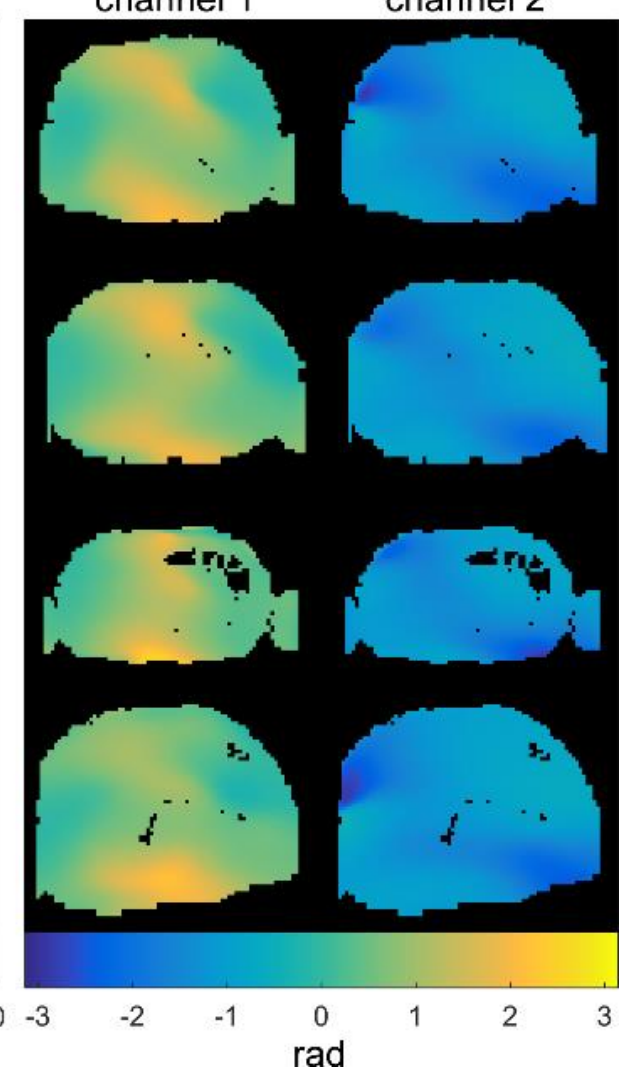

Figure 1 : Examples illustrating the inter-subject variability of transmit $\boldsymbol{B}_{1}$ field in the abdomen for four subjects (top to bottom). a. $B_{1^{+}}$magnitude for both transmit channels. $\boldsymbol{b} . \mathrm{B}_{1}{ }^{+}$phase. Field of view and windowing are identical for all subjects.

\section{THEORY}

In this section, we briefly present subject-specific (tailored) and universal $\mathrm{kT}_{\mathrm{T}}$ points pulse design. Then we introduce the clustering and pulse design specificities of SmartPulse. 


\subsection{Tailored $\mathrm{K}_{\mathrm{T}-\text { Points Pulse Design }}$}

The $\mathrm{kT}_{\mathrm{T}}$-points pulse design consists in homogenizing the flip angle (FA) distribution in a region of interest $(\mathrm{ROI})$ by optimising simultaneously RF complex coefficients, k-space locations (32) and durations (33) of each kt-point sub-pulse. With $N_{k} T$ sub-pulses on a pTx system equipped with $N_{C h}$ transmission channels, and using the vectors $\mathbf{x}, \mathbf{k}$ and $\mathbf{t}$ to represent, respectively, all sub-pulse RF complex coefficients, three-dimensional k-space locations, and sub-pulse durations, the optimization problem can be expressed as $(22,34)$ :

$$
\underset{\mathbf{x}, \mathbf{k}, \mathbf{t}}{\arg \min } \frac{1}{\alpha_{T} \sqrt{N_{v}}}\left\|\mathrm{~A}(\mathbf{x}, \mathbf{k}, \mathbf{t})-\alpha_{T} I\right\|_{2}, \quad(\mathbf{x}, \mathbf{k}, \mathbf{t}) \in \mathbb{C}^{N_{C h} N_{k T}} \times \mathbb{R}^{3 N_{k T}} \times \mathbb{R}^{N_{k T}}
$$

where $\alpha_{T}$ is the targeted FA (a scalar), $l$ is the identity vector spanning the $N_{V}$ voxels in the $\mathrm{ROI}$, and $\mathrm{A}$ is the Bloch operator returning a FA for each voxel depending on its $\mathrm{B}_{1}{ }^{+}$and $\Delta \mathrm{f}_{0}$ values. Optimization is performed under peak power, average power, and global and local specific absorption rate (SAR) constraints (35) to comply with hardware limits and patient safety.

\subsection{Universal $\mathrm{K}_{\mathrm{T}}$-Points Pulse Design}

The design of a universal pulse (22) seeks to homogenise excitation simultaneously over the whole population, based on a sample of $N_{S}$ subjects. In this work, the approach is a compromise between refs (22) and (23): in order to reduce the global FA inhomogeneity in the whole population while avoiding outliers, the mean plus standard deviation of the normalised root-mean-square FA errors (NRMSE) achieved in each subject was chosen as the cost function to be minimized. If we represent by $\mathcal{A}=\left\{\mathrm{A}_{1}, \ldots, \mathrm{A}_{N_{S}}\right\}$ the set of Bloch operators associated with each of the $N_{S}$ subjects in the population, we solve the following problem:

$$
\underset{\mathbf{x}, \mathbf{k}, \mathbf{t}}{\arg \min }(\mathrm{M}(\boldsymbol{\epsilon})),(\mathbf{x}, \mathbf{k}, \mathbf{t}) \in \mathbb{C}^{N_{C h} N_{k T}} \times \mathbb{R}^{3 N_{k T}} \times \mathbb{R}^{N_{k T}}
$$

Where $\boldsymbol{\epsilon}=\left(\epsilon_{S}\right)_{1 \leq s \leq N_{S}}$ is the vector gathering the NRMSE of each subject, defined by $\epsilon_{S}=\frac{1}{\alpha_{T} \sqrt{N_{v, s}}}\left\|\mathrm{~A}_{S}(\mathbf{x}, \mathbf{k}, \mathbf{t})-\alpha_{T} I\right\|_{2}$, and $\mathrm{M}(\boldsymbol{\varepsilon})=\langle\mathcal{E}\rangle+\mathrm{SD}(\mathcal{E}) .\langle\cdot\rangle$ and $\mathrm{SD}(\cdot)$ represent the sample mean and the sample standard deviation operators, respectively. Without any additional difficulty, this problem is also solved under all strict constraints as described in the tailored case. 


\subsection{SmartPulse Design Process}

The SmartPulse design process is divided into (i) a clustering and pulse design step, and (ii) a classification step. The aim of the clustering and pulse design step is to partition the database into "homogeneous" clusters and to design for each cluster a universal kT-points pulse. The classification step consists in training a machine learning algorithm to assign the most appropriate pulse to new subjects, given a set of simple features that do not require additional calibration - in particular without knowledge of $\mathrm{B}_{1}{ }^{+}$and $\Delta \mathrm{f}_{0}$ distributions. In the following, we provide the formalism of the clustering process.

For each of the $N_{S, 0}$ subjects of the database, we design one tailored kT-points pulse (minimization of the objective given by Eq. [1]). Each of the $N_{S, 0}$ tailored kTpoints pulses is then applied on every subject of the database and the resulting FA NRMSEs are stored in the matrix:

$$
\mathcal{E}=\left(\epsilon_{s, p}\right)_{1 \leq s, p \leq N_{S, 0}}
$$

where $\epsilon_{s, p}$ denotes the NRMSE achieved by the $p^{\text {th }}$ pulse when applied to the $s^{\text {th }}$ subject. By defining subject vectors $\epsilon_{s}=\left(\epsilon_{s, p}\right)_{1 \leq p \leq N_{S, 0}}$ (i.e. the $p^{\text {th }}$ column of $\mathcal{E}$ ), we compute $\mathcal{D}$, the distance matrix between subjects:

$$
\mathcal{D}=\left(\left\|\boldsymbol{\epsilon}_{\boldsymbol{s}}-\boldsymbol{\epsilon}_{\boldsymbol{s}^{\prime}}\right\|_{2}\right)_{1 \leq s, s^{\prime} \leq N_{S, 0}}
$$

This distance matrix is now exploited to partition the database into a desired number of clusters. To do so, we perform agglomerative hierarchical clustering on $\mathcal{D}$ with complete linkage (36), i.e. with the distance between two clusters of subjects $/$ and $J$ defined as

$$
d(I, J)=\max _{\boldsymbol{\epsilon}_{\mathbf{s}} \in I, \boldsymbol{\epsilon}_{\mathbf{s}^{\prime}} \in J}\left\|\boldsymbol{\epsilon}_{\mathbf{s}}-\boldsymbol{\epsilon}_{\mathbf{s}^{\prime}}\right\|_{2}
$$

It is a bottom-up process that starts with each individual subject forming a singleton cluster. A new cluster $K$ is created by joining the two clusters $/$ and $J$ of minimal pairwise distance $d(I, J)$; I and $J$ are then removed from the set. Clusters are iteratively joined two by two to form new larger clusters, until a single cluster containing all subjects is obtained. This process both leverages and represents all the distances between individuals and between groups of subjects. It emphasizes the inherent structure of the set of subjects, and allows to group subjects that display similar behaviour to various RF pulses in terms of NRMSE. 
Finally, given a number of desired clusters $N_{C}$, we stop the merging process to reach this exact number. If we denote by $\mathcal{C}_{j}$ the set of indices of subjects belonging to the $f^{\text {th }}$ cluster, clustered pulse design boils down to solving Eq. [2] for each subset $\mathcal{A}_{j}$ :

$$
\mathcal{A}_{j}=\left\{\mathrm{A}_{i} \mid i \in \mathcal{C}_{j}\right\}, j \in\left[1 . . N_{C}\right]
$$

to obtain $N_{C}$ universal $\mathbf{k}_{\mathrm{T}}$-points pulses $\mathbf{p}_{\mathbf{j}}=\left(\mathbf{x}_{\mathbf{j}}, \mathbf{k}_{\mathbf{j}}, \mathbf{t}_{\mathbf{j}}\right)$.

\section{Methods}

\subsection{Subjects and Scanner}

Data acquired for a previous study (10) on 50 consecutive subjects were reused here as the clustering set: it corresponds to subjects 1 through $N_{S, 0}=50$. For the present work, measurements were performed over a three-week period on $N_{S, 1}=$ 30 additional subjects, thus forming a training population of $N_{\text {train }}=80$ subjects.

Finally, a testing population of $N_{\text {test }}=53$ consecutive subjects was acquired over four weeks. A total of 133 patients (69 men, 64 women, age: 22-89 years, height: 1.45$1.91 \mathrm{~m}$, weight: $45-140 \mathrm{~kg}, \mathrm{BMI}: 17.6-43.7 \mathrm{~kg} \cdot \mathrm{m}^{-2}$ ) were therefore included. 23 subjects from the testing population underwent DCE-MRI while in the presence of one of the authors (R.T.-T.), over the last two weeks of the study; they constitute the imaging population. The different subject populations are represented in Figure 2.
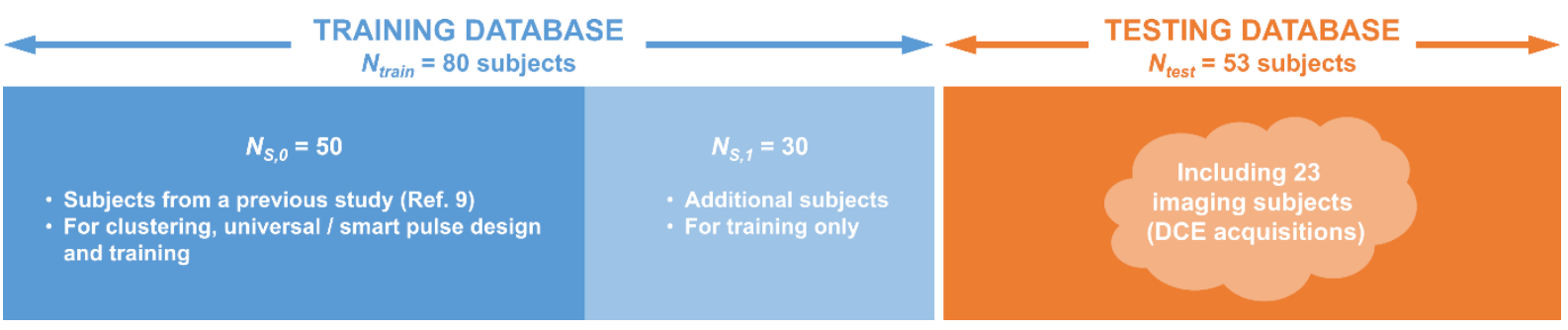

Figure 2 : Visualization of the different subject populations used for training or testing the SmartPulse method. Some subjects from the training database were also used for clustering and pulse design. 23 subjects from the testing population received DCE-MRI while a pulse designer was present and formed the imaging population: images obtained with different techniques before and after injection were compared. 
Our study was approved by the institutional review board of Centre Hospitalier Universitaire Henri Mondor; informed consent was waived. Acquisitions were carried out on a MAGNETOM Skyra (Siemens Healthcare GmbH, Erlangen, Germany) 3T scanner, equipped with a two-channel pTx system used in clinical routine. Two 30channel coil arrays (anterior and posterior) were used for reception.

\section{2. $\mathrm{B}_{1}{ }^{+}$and Off-Resonance Mapping}

$\mathrm{B}_{1}+$ maps were measured through a manufacturer automatic adjustment procedure, a free-breathing magnetization-prepared turbo FLASH sequence performed in about 30 seconds. They were the same maps as the ones used for patient-specific static RF shimming: $80^{\circ}$ saturation, $8^{\circ}$ excitation, $300-\mathrm{ms}$ preparation time, $\mathrm{TR}=5.0 \mathrm{~s}, 64 \times 64$ matrix, $7.0 \times 7.0 \mathrm{~mm}^{2}$ in-plane resolution, 326 -mm-thick slices.

$\Delta \mathrm{f}_{0}$ maps were needed for pulse design and FA simulations per se, but also to discriminate between water and fat voxels. Indeed, the pulses were intended for a fat-suppressed sequence, so only water voxels were considered for FA homogenization, leading to increased pulse performance in water as well as lower computation times. Assuming tissues do not mix, this was achieved with a two-echo FLASH acquisition acquired in one breath-hold $(10 \mathrm{~s})$ with $\triangle \mathrm{TE}=0.95 \mathrm{~ms}, 96 \times 96$ matrix, $4.0 \times 4.0 \mathrm{~mm}^{2}$ resolution, 464.2 - $\mathrm{mm}$-thick slices. Supporting Information Figure $\mathrm{S} 1$ depicts a typical transverse slice from a $\Delta \mathrm{f}_{0}$ abdominal map with its corresponding histogram, from which pure fat can be excluded.

Pulse design was performed from a 3D mesh of 5-mm isotropic voxels, so both $\mathrm{B}_{1}{ }^{+}$and $\Delta \mathrm{f}_{0}$ maps were resampled to match that resolution.

\subsection{Pulses Compared}

For each subject of the testing population (Subjects 81 through 133), six pulse types were used:

- pтF: TrueForm, the scanner default pre-set elliptically polarised pulse;

- ptss: manufacturer's patient-tailored static RF shimming with coefficients automatically calculated by the scanner based on the subject's $B 1^{+}$maps, but with a maximum voltage constrained to be less than or equal to that of TrueForm; 
- рткт: patient-tailored kT-points dynamic RF shimming, computed offline;

- pukт: universal kt-points;

- psP: SmartPulse kT-points.

Duration of hard pulses PTF and pTss was set to $100 \mu \mathrm{s}$. Pulse design for other techniques is described below.

\subsubsection{Tailored and Universal $\mathrm{K}_{\mathrm{T}}$-Points Pulse Design}

9-kT-point tailored pulses (рткT) were designed, under SAR and hardware constraints, as done in ref. (10). With the goal of using them for DCE-MRI, a FA of $\alpha_{T}=11^{\circ}$ was targeted, for a TR of $6 \mathrm{~ms}$, and a $1300-\mu$ s pulse duration limit was set. For local SAR prediction, virtual observation points (VOP) (37) provided by the scanner for each of the subjects were used. It is assumed that the vendor performed VOP computations accounting for body models that include a wide range of anatomical variations, thus admissibly covering the most unfavorable cases in terms of SAR through the population studied in the present work.

A universal pulse was also designed on Subjects 1 through 50, according to Eq. [2]. The pulse used throughout this study, puкт, had five sub-pulses and was 770microsecond-long. This reduction in the number of sub-pulses as compared to the tailored pulses was empirically found as the right compromise between the number of degrees of freedom necessary to yield sufficiently uniform FA profiles and short pulse duration. The latter criterion indeed is important to accommodate for large $\Delta f_{0}$ variations across the population.

All pulses were designed using the active-set constrained optimization algorithm from MATLAB R2016a (The Mathworks, Natick, MA) on a laptop computer (Intel Core i7-4712HQ CPU, NVIDIA Quadro K1100m GPU).

\subsubsection{SmartPulse Clustering and Pulse Design}

Clustering and SmartPulse design were based on Subjects 1 through $N_{s, 0}=$ 50, as pictured in Figure 3, where matrices $\mathcal{E}$ and $\mathcal{D}$ (Eq. 3 and 4), obtained using tailored $\mathrm{k}_{\mathrm{T}}$-points described above, are shown both before and after clustering. 

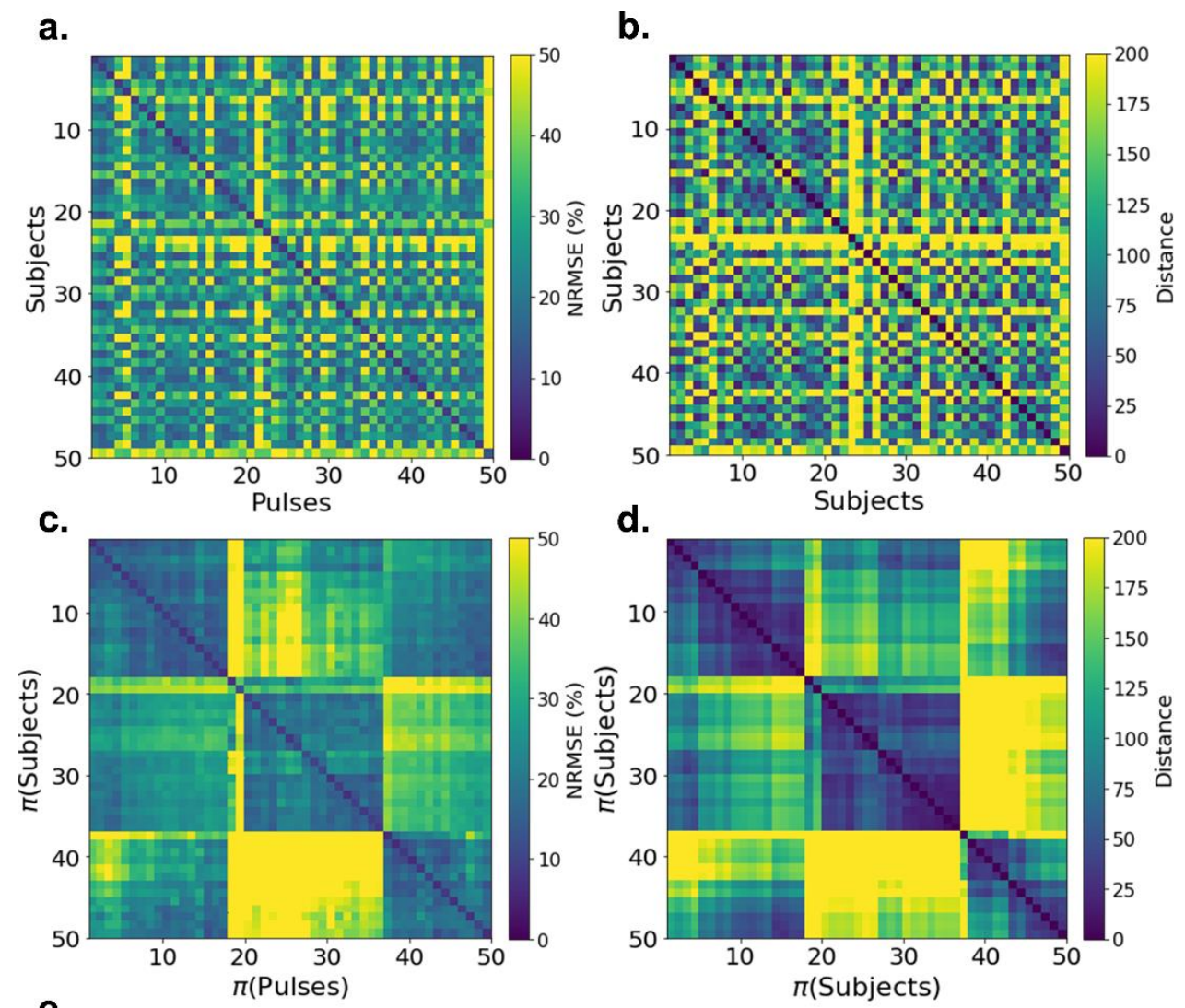

e.

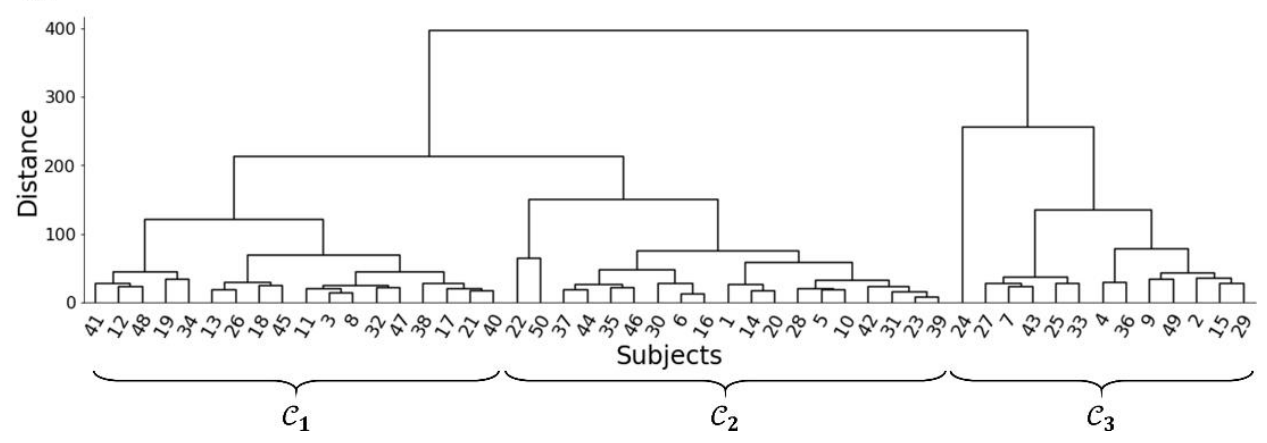

Figure 3 : Creation of three clusters from subjects 1 through 50. a. Matrix $\mathcal{E}$ (Eq. [3]) of NRMSEs. b. Matrix $\mathcal{D}$ of subject pairwise distance (Eq. [4]). c-d. Reorganised matrices $\mathcal{E}$ and $\mathcal{D}$ (respectively) using permutation $\pi$ output by agglomerative hierarchical clustering applied on $\mathcal{D}$. Cluster structure becomes visible. e. Dendogram output by agglomerative clustering applied on $\mathcal{D}$. Definition of $\mathcal{C}_{1}, \mathcal{C}_{2}$ and $\mathcal{C}_{3}$, the three clusters used for SmartPulse pulse design in this study.

Agglomerative hierarchical clustering was done using Python v2.7 and the fastcluster package (36). Given the relatively small number of training data, a limited number of clusters (three) was chosen. Subject 24 behaved as an outlier and formed its own cluster, so it was manually assigned to cluster $\mathcal{C}_{3}$ (Figure $3 e$ ). 
Pulses SmartPulse 1 (psp,1), SmartPulse 2 (psp,2) and SmartPulse 3 (psp,3) were created for cluster $\mathcal{C}_{1}, \mathcal{C}_{2}$ and $\mathcal{C}_{3}$ respectively, with the same 5-kT-point design parameters as for рuкт. Their optimized durations were $700 \mu \mathrm{s}, 720 \mu \mathrm{s}$ and $690 \mu \mathrm{s}$, respectively. Like Universal Pulses, SmartPulses have fixed RF amplitudes and were applied as such to every subject without voltage scaling.

\subsection{SmartPulse Subject Classification}

Ten features likely to influence abdominal composition and geometry, and therefore $\mathrm{B}_{1}{ }^{+}$distribution, were used for classification. These features were all extracted from data from the localizer (Figure 4), which is a compulsory sequence placed at the very beginning of the protocol and used to set up the field of view (FOV) of subsequent acquisitions. The localizer sequence used locally for liver imaging consists in 5 axial, 7 coronal and 11 sagittal T1-weighted 7.0-mm-thick slices of 0.8-mm in-plane resolution, all acquired in one breath-hold ( $\mathrm{TA}=17 \mathrm{~s})$. Table 1 gathers the features corresponding to the subjects shown in the figures of this study, as well as a population summary. Subject's age, sex $(38,39)$, weight and height, given by the patients during registration, are compulsory data for any MRI examination on this scanner, and are available as DICOM fields, as specified in PS3.6: Data Dictionary (40). The global SAR measured by the machine during the localizer acquisition was also retrieved from DICOM fields. Subject's body mass index (BMI) was derived from height and weight. Additional manufacturer-specific metadata was available, among which the reference voltage, calibrated by the scanner for each subject, and defined as the voltage necessary for a 500- $\mu$ s hard pulse to presumably create $90^{\circ}$ average excitation in a $1-\mathrm{cm}$-thick transversal slice at magnet isocenter (41). In the default transmit configuration, this value is related to the $\mathrm{B}_{1}+$ distribution within this slice. The subject's abdominal width and height (2 features) were estimated by fitting an ellipse inscribed in an axial slice, and retrieving its major and minor axes (respectively). The most inferior slice was picked to ensure the ellipse fitting was performed in the abdomen, not in the thorax. In this pilot study, however, for some patients, the localizer was really off-centered; in those cases, the ellipse fitting was performed on the central slice of the magnitude images associated with the $\Delta f_{0}$ map. One last feature was derived from these measurements: the abdominal ratio (height over width). 
Table 1. Features extracted from the five subjects focused on in this study

\begin{tabular}{|c|c|c|c|c|c|c|c|c|c|c|}
\hline $\begin{array}{l}\text { Subject } \\
\text { number }\end{array}$ & $\begin{array}{l}\text { Age } \\
\text { years }\end{array}$ & Sex & $\begin{array}{c}\text { Height } \\
\quad m\end{array}$ & $\begin{array}{c}\text { Weight } \\
\text { kg }\end{array}$ & $\begin{array}{l}\text { BMI } \\
\mathrm{kg} \cdot \mathrm{m}^{-2}\end{array}$ & $\begin{array}{c}\text { Abdomen } \\
\mathrm{APa}^{\mathrm{a}} \\
\mathrm{mm}\end{array}$ & $\begin{array}{c}\text { Abdomen } \\
\mathrm{LR}^{\mathrm{a}} \\
\mathrm{mm}\end{array}$ & $\begin{array}{c}\text { Abdomen } \\
\text { ratio }^{\mathrm{a}} \\
\%\end{array}$ & $\begin{array}{c}V_{\text {ref }^{b}} \\
V\end{array}$ & $\begin{array}{c}\text { Localizer } \\
\mathrm{SAR}_{\mathrm{g}}^{\mathrm{c}} \\
W \cdot \mathrm{kg}^{-1}\end{array}$ \\
\hline 110 & 62 & $M$ & 1.76 & 119 & 38.4 & 338 & 408 & 83 & 494 & 0.064 \\
\hline 113 & 40 & $F$ & 1.65 & 65 & 23.9 & 198 & 285 & 69 & 430 & 0.075 \\
\hline 115 & 58 & $M$ & 1.80 & 85 & 26.2 & 232 & 333 & 70 & 633 & 0.099 \\
\hline 127 & 65 & $M$ & 1.70 & 79 & 27.3 & 237 & 353 & 67 & 571 & 0.087 \\
\hline 132 & 48 & M & 1.80 & 90 & 27.8 & 282 & 363 & 78 & 460 & 0.070 \\
\hline All $N_{\text {test }}{ }^{d}$ & $\begin{array}{c}53 \\
(16)\end{array}$ & $\begin{array}{l}M: 26 \\
F: 27\end{array}$ & $\begin{array}{c}1.68 \\
(0.09)\end{array}$ & $\begin{array}{c}78 \\
(22)\end{array}$ & $\begin{array}{l}27.4 \\
(6.3)\end{array}$ & $\begin{array}{l}242 \\
(36)\end{array}$ & $\begin{array}{l}332 \\
(38)\end{array}$ & $\begin{array}{c}73 \\
(4.8)\end{array}$ & $\begin{array}{l}509 \\
(75)\end{array}$ & $\begin{array}{c}0.078 \\
(0.016)\end{array}$ \\
\hline
\end{tabular}

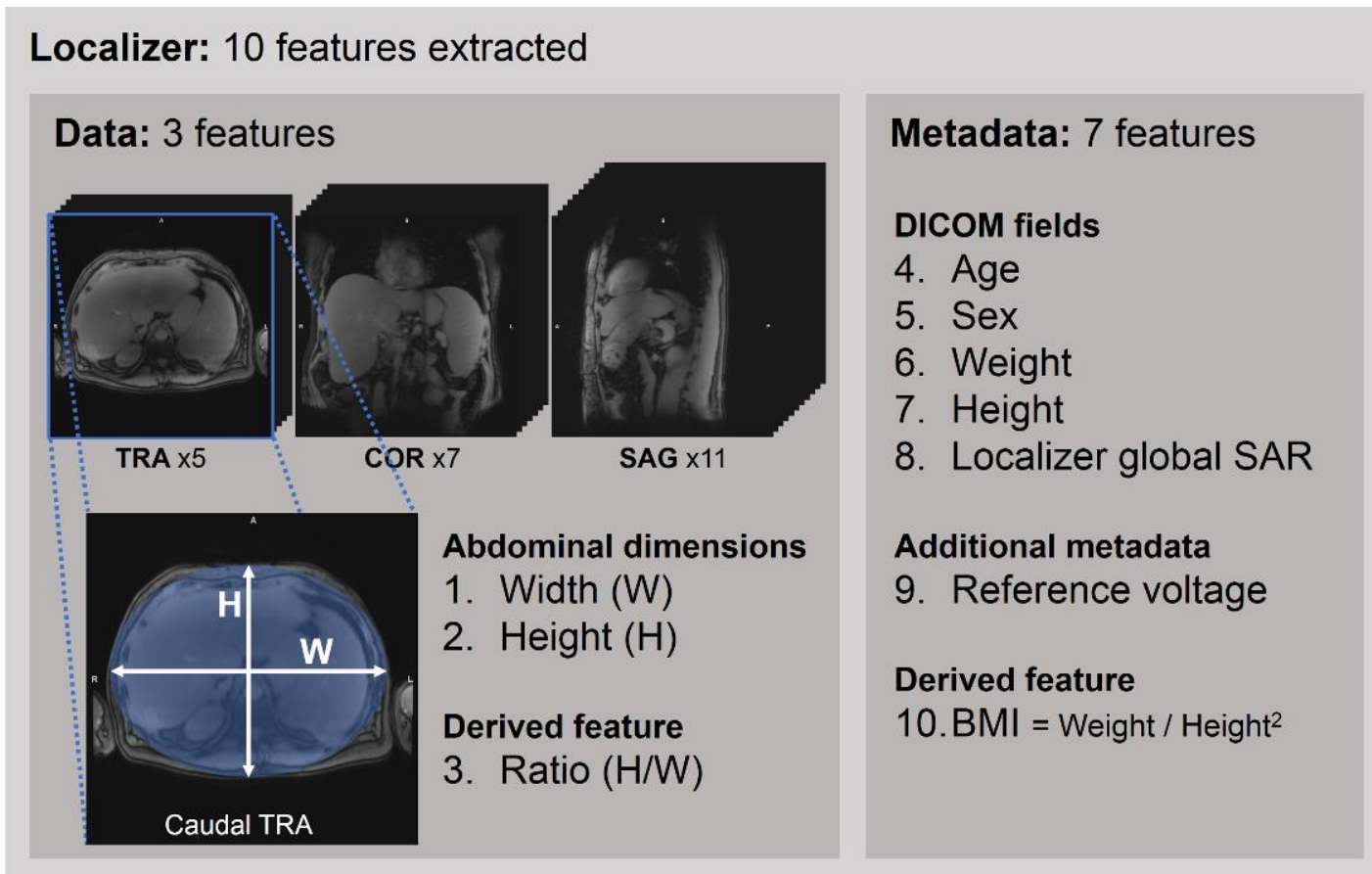

Figure 4 : Features extracted from a "localizer" acquisition (compulsory sequence at the beginning of any protocol). Abdomen dimensions were measured by fitting an ellipse in one axial slice; more slices could be analysed with the help of automated patient and field of view positioning, allowing finer patient characterization. Additionally and with some post-processing, coronal and sagittal views could provide useful liver and spleen geometry information. TRA/COR/SAG= transverse/coronal/ sagittal planes; $S A R=$ specific absorption rate; $B M I=$ body mass index; reference voltage $=$ voltage required for a $500-\mu$ s hard pulse to perform $90^{\circ}$ excitation. 
The classification algorithm was implemented using Scikit-learn (42) and trained on the population of $\mathrm{N}_{\text {train }}=80$ subjects, among which where the 50 database subjects used to create psp,1-3. Subjects from the clustering set were naturally labelled with the cluster they belong to. For the $N_{S, 1}=30$ additional training subjects, the effect of all three SmartPulses was simulated, and each subject was labelled according to the pulse yielding the lowest NRMSE. An extremely randomized trees algorithm (43) with 4000 trees and a support vector machine multiclass classifier $(44,45)$ with a radial basis function kernel were joined by Scikit-learn's "soft" vote and tuned by repeated stratified cross-validation on this set with 50 shuffles and 5 splits. Given that the extremely randomized trees and the nonlinear support vector machine algorithms accommodate redundant and/or uninformative features and given the relatively low dimensionality (10 features) as compared to the number of observations ( 80 subjects) of the problem, no feature selection was performed. Analysis of the importance of the features, provided in Supporting Information Table $\mathrm{S} 1$, further validates this choice; additionally, comparison with a "dummy" feature shows that all included features had an impact on the classification. Cross-validation accuracy was $85 \%$.

To assess classification performance, a prediction accuracy score was calculated on the test set, i.e. the proportion of subjects who were assigned the pulse yielding minimal NRMSE. A relaxed accuracy score was also determined, which regarded subjects as misclassified only if the NRMSE associated with their assigned pulse was $2 \%$ above the lowest possible.

Finally, the time needed for feature extraction and pulse prediction on individual subjects from the test population was measured.

\subsection{Excitation Homogeneity}

FA maps obtained with each technique were simulated by numerical integration of Bloch's equations based on actual $B_{1}{ }^{+}$and $\Delta f_{0}$ maps measured in subjects. FA average, coefficient of variation (CV) and NRMSE over water voxels in the volume of interest were estimated.

In the SmartPulse case, all three pulses were tested, and the one yielding the lowest NRMSE was defined as PSP,ideal, and used to assess the performance of an "ideal" process with no prediction errors. 
Matched-pair Wilcoxon signed-rank tests were computed using the SciPy (46) stats package whenever FA NRMSE, CV or means of different techniques were compared. Reported $p$-values are two-sided significance levels; null hypothesis of equality of distribution medians was rejected for $p$ less than 0.05 .

\subsection{In Vivo Acquisitions}

Additional sequences were run on subjects from the imaging population to compare images obtained with PTF, PTSS, PTKT and the predicted psP. To avoid disturbing the diagnostic DCE-MRI protocol, those sequences were only inserted prior to contrast agent injection and in late phase (Supporting Information Figure S2), similarly to what was done in Ref. (10).

The sequence used was a T1-weighted 3D FLASH, with "quick fat-saturation" (47) to remove hypersignal due to the short $T_{1}$ of fat that could mask contrast-agentrelated enhancement. Fat saturation was achieved with a fat-selective $90^{\circ}$-pulse of Gaussian shape in the TrueForm transmission mode, identical in all sequences. Sequence parameters were: $\mathrm{FA}=11^{\circ}, \mathrm{TR} / \mathrm{TE}=6 / 3 \mathrm{~ms}, 320 \times 220 \times 72$ matrix, $1.2 \times 1.2 \times 3.5 \mathrm{~mm}^{3}$ resolution, GRAPPA factor 2 in the phase encoding direction (anterior-posterior), $80 \% / 50 \%$ phase/slice resolution, partial Fourier factor of $6 / 8$, $505 \mathrm{~Hz} /$ pixel bandwidth. Acquisition time was less than 23 seconds. The matrix size and/or resolution was sometimes adjusted to accommodate larger patients while ensuring an acquisition time compatible with a breath-hold. Up to $33 \%$ oversampling was needed in the partition-encoding direction to avoid aliasing. Two 30-channel surface coils (anterior and posterior) were used for reception. Only the channels necessary to cover the FOV were selected; this was done automatically by the scanner, and allowed to keep oversampling to a minimum. The manufacturer's "Prescan Normalize" procedure was applied to all series in order to correct images for reception profile. However, this technique assumes homogeneous body coil reception, which is not the case in the abdomen as the corresponding reception profile is also affected by the $B_{1}$ artifact; some inhomogeneity may therefore remain.

Elastic registration was applied to all acquisitions using Siemens software. This allowed to calculate, for each technique, the contrast enhancement (CE) and the enhancement ratio (ER) defined as: 


$$
\mathrm{CE}=\mathrm{S}_{\text {late }}-\mathrm{S}_{\text {ref }}
$$

and

$$
\mathrm{ER}=\frac{\mathrm{CE}}{\mathrm{S}_{\mathrm{ref}}} \times 100 \%
$$

where Sref and Slate represent signal before and after injection, respectively. ER presents the advantage of completely removing the reception profile; however, it will artificially increase with FA overshoot, contrarily to CE.

\section{Results}

\subsection{SmartPulse Subject Classification}

Once the localizer sequence was acquired, the full process of feature extraction and SmartPulse prediction for one subject systematically took less than two seconds. Strictly speaking, the accuracy of the algorithm on test data was $74 \%$. However, in many cases two pulses would perform similarly well, so the second best pulse may not necessarily be regarded as a "wrong" option. This is illustrated on Figure $5 a$ where, for each testing subject, coloured circles represent the NRMSE of the different psp options; filled circles correspond to the actual prediction. Relaxed accuracy (with a $+2 \%$ NRMSE tolerance) was $81 \%$. This is to be compared with the chance level for a three-class problem, namely $33 \%$.

\subsection{Excitation Homogeneity}

Figure 5b compares SmartPulse to TrueForm, the default calibration-free pulse provided by the vendor. Universal pulse performance is also indicated. Considering the $+2 \%$ tolerance, predicted psP yielded lower NRMSE than PTF or was at least the best available psp for 46 subjects (87\%). Supporting Information Figure S3 gathers individual NRMSE results for all tested pulse designs. psp performed consistently better than PTF, PTss and PUKT. All were unsurprisingly outperformed by PTKT. 
a.

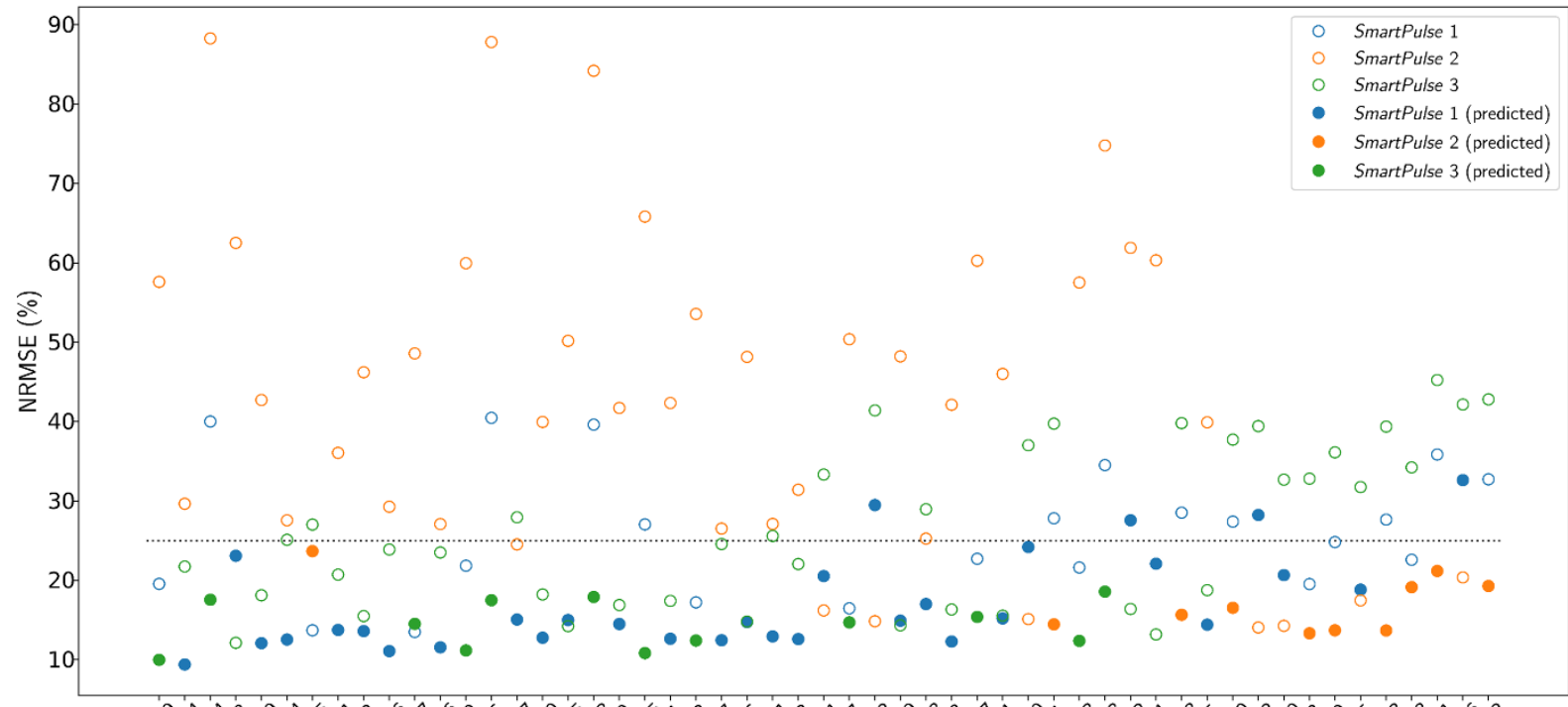

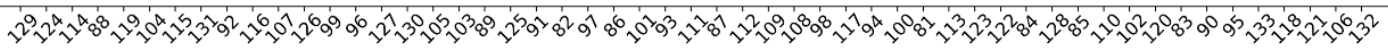

b.

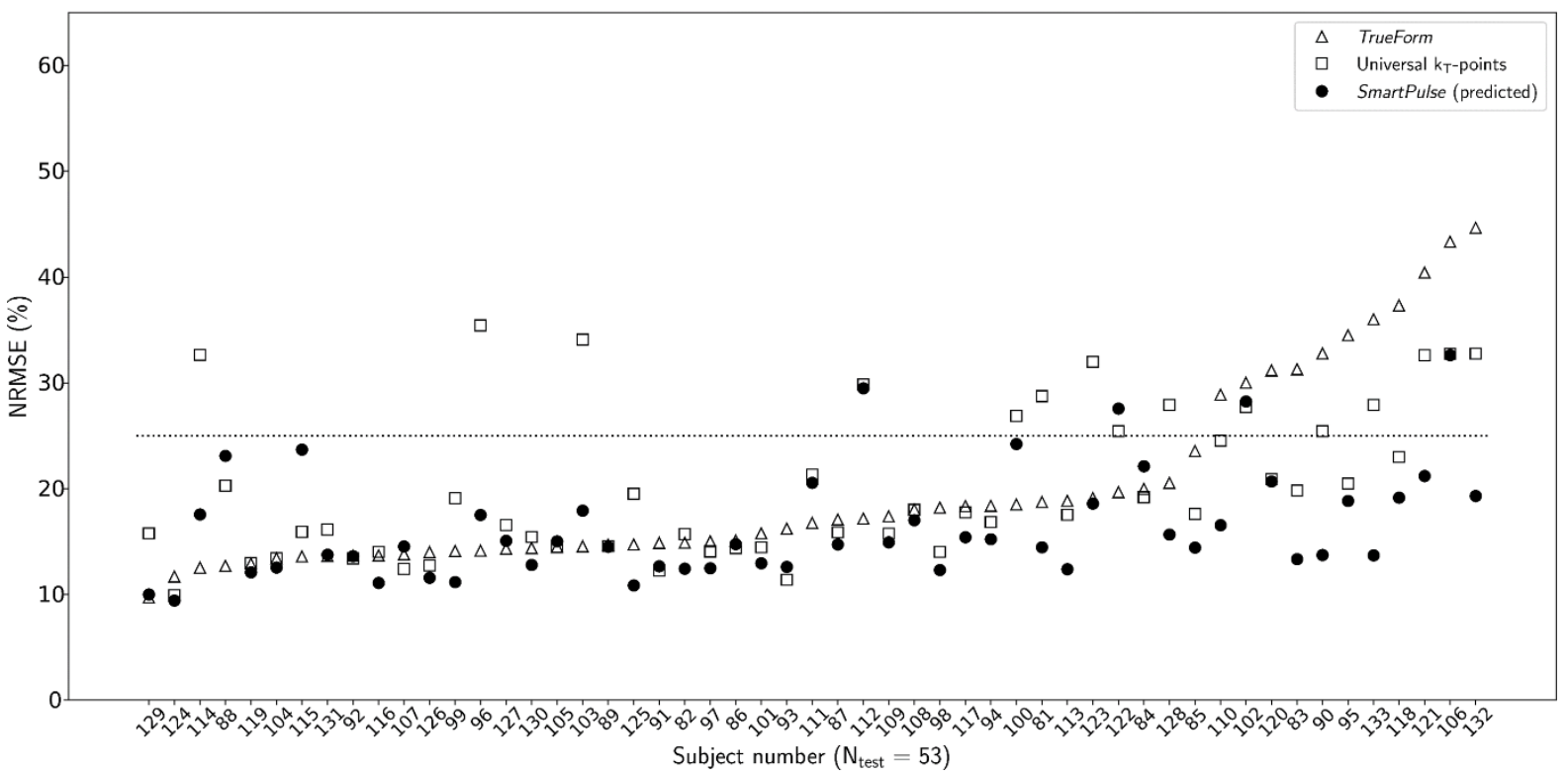

Figure 5 : NRMSE simulation results for all test subjects, sorted in increasing TrueForm inhomogeneity. a. Performance of predicted SmartPulse (filled circles) compared to other SmartPulse options (hollow circles). Colors render the three different possible SmartPulse classes. For instance, for subject \#129 (first entry), pSP $=p_{S P, \text { ideal }}=$ SmartPulse 3, while for subject \#88 (fourth entry), $p_{S P}=$ SmartPulse 1 and $p_{S P, \text { ideal }}=$ SmartPulse 3. b. Comparison of TrueForm, Universal and SmartPulse pulse designs. For each subject, only the predicted SmartPulse is shown, not all three possibilities. The dashed line indicates 25\% NRMSE. Notice how subjects with a high TrueForm NRMSE are brought towards more acceptable values. 
In addition, psp allowed $93 \%$ of subjects to get a NRMSE below $25 \%$ (dashed line on Figure 5), which is much more than рuкт (72\% of subjects), pтss $(77 \%)$ and PTF (79\%), but less than рTKT (100\%). However, psp,ideal would also have allowed $100 \%$ of subjects below that threshold. Yet, in three cases, even the optimal SmartPulse (psp,ideal) gave a lower performance than TrueForm: subject 96 (18\% NRMSE for PSP,ideal, $14 \%$ for pTF), subject 103 (18\% vs $15 \%$ ) and subject 114 (18\% vs $13 \%)$.

Sample average and SD of NRMSE, CV and mean of FA are available in Table 2, with more detail on their distribution in Figure 6. Average psp NRMSE was $16 \%$ (SD: $5 \%$ ), lower than that of pTF, pTss and pukT, respectively 20\% (SD: $9 \%$, $p=0.002$ ), $21 \%$ (SD: $10 \%, p=0.0005$ ) and 20\% (SD: $5 \%, p<0.0001$ ). Other metrics allow deeper understanding of pulse performance: FA CV expresses sheer homogeneity, while FA average measures a possible FA bias as compared to the nominal value. With a mean CV of $13 \%$, psp outperformed all pulses but рткт. Although pTss yielded better homogeneity than $\mathrm{PTF}_{\mathrm{TF}}(p<0.0001)$, it drifted further away from the FA target average: $9.6^{\circ}\left(\mathrm{SD}: 1.3^{\circ}\right)$ and $9.3^{\circ}\left(\mathrm{SD}: 1.3^{\circ}\right)$ respectively $(p<0.0001)$. This was not the case for psp, which achieved better performance on FA average as well, with $10.7^{\circ}\left(\mathrm{SD}: 1.3^{\circ}\right)$, and even $10.8^{\circ}\left(\mathrm{SD}: 0.8^{\circ}\right)$ for psp,ideal $(p<0.0001$ in both cases).

Finally, psp was not too energy-demanding, with an average local SAR across the test population lying at $21 \%$ (SD: $4 \%$ ) of the maximal authorized value. One may be concerned about implications of mispredictions in terms of SAR, but SmartPulse seems to behave favourably in this respect. Indeed, out of the 53 test subjects, the highest SAR encountered was 37\%, considering all three psp options.

\subsection{In Vivo Acquisitions}

Figures 7 and 8, and Supporting Information Figure S4 show examples of images acquired before contrast agent injection and in late phase, with CE and ER maps calculated according to Eq. [6] and [7]. 
Table 2 : Detailed homogeneity assessment results of all tested pulses for the five subjects from Table 1.

\begin{tabular}{|c|c|c|c|c|c|c|c|}
\hline \multirow[b]{2}{*}{ Subject } & \multicolumn{7}{|c|}{ Flip Angle NRMSE [\%] } \\
\hline & PTF & ptss & potss & PTKT & PUKT & PSP & PSP,ideal $^{\mathbf{a}}$ \\
\hline 110 & 29 & 32 & 15 & 8 & 25 & 17 & 17 \\
\hline 113 & 19 & 20 & 14 & 8 & 18 & 12 & 12 \\
\hline 115 & 14 & 13 & 13 & 7 & 16 & 23 & 14 \\
\hline 127 & 14 & 14 & 12 & 7 & 17 & 15 & 15 \\
\hline 132 & 45 & 49 & 26 & 9 & 33 & 19 & 19 \\
\hline \multirow[t]{2}{*}{ All $N_{\text {test }}{ }^{\mathrm{b}}$} & $20(9)$ & $21(10)$ & $14(4)$ & $8(2)$ & $20(5)$ & $16(5)$ & $14(3)$ \\
\hline & \multicolumn{7}{|c|}{ Flip Angle Coefficient of Variation [\%] } \\
\hline Subject & PTF & ptss & Potss & PTKT & PUKT & PsP & PSP,idealPSP, best $^{\mathrm{a}}$ \\
\hline 110 & 18 & 14 & 15 & 8 & 14 & 17 & 17 \\
\hline 113 & 15 & 15 & 14 & 8 & 12 & 12 & 12 \\
\hline 115 & 14 & 13 & 13 & 7 & 14 & 14 & 10 \\
\hline 127 & 14 & 13 & 12 & 7 & 13 & 11 & 11 \\
\hline 132 & 28 & 27 & 27 & 9 & 27 & 17 & 17 \\
\hline \multirow[t]{2}{*}{ All $N_{\text {test }} \mathrm{b}$} & $16(4)$ & $15(4)$ & $14(4)$ & $8(2)$ & $14(4)$ & $13(3)$ & $13(2)$ \\
\hline & \multicolumn{7}{|c|}{ Flip Angle Average [degrees] } \\
\hline Subject & PTF & ptss & Potss & PTKT & PUKT & PsP & psP,best $^{a}$ \\
\hline 110 & 8.2 & 7.7 & 10.4 & 10.9 & 8.6 & 10.5 & 10.5 \\
\hline 113 & 9.5 & 9.3 & 10.8 & 10.9 & 12.2 & 10.4 & 10.4 \\
\hline 115 & 10.8 & 10.4 & 10.8 & 10.9 & 9.9 & 12.9 & 9.9 \\
\hline 127 & 10.6 & 10.3 & 10.8 & 10.9 & 9.7 & 9.8 & 9.8 \\
\hline 132 & 6.4 & 5.9 & 10.0 & 10.9 & 8.1 & 9.7 & 9.7 \\
\hline All $N_{\text {test }}{ }^{b}$ & $\begin{array}{c}9.6 \\
(1.3)\end{array}$ & $\begin{array}{c}9.3 \\
(1.3)\end{array}$ & $\begin{array}{l}10.7 \\
(0.2)\end{array}$ & $\begin{array}{l}10.9 \\
(0.0)\end{array}$ & $\begin{array}{l}10.7 \\
(1.8)\end{array}$ & $\begin{array}{l}10.7 \\
(1.3)\end{array}$ & $\begin{array}{l}10.8 \\
(0.8)\end{array}$ \\
\hline
\end{tabular}

$\mathrm{p}_{\mathrm{TF}}=$ TrueForm; $\mathrm{p}_{\mathrm{TSS}}=$ patient-tailored static RF shimming calculated by the scanner; $\mathrm{p}_{\mathrm{OTSS}}=$ optimal patient-tailored static RF shimming; $\mathrm{p}_{\mathrm{TKT}}=$ tailored $\mathrm{k}_{\mathrm{T}}$-points; $\mathrm{p}_{\mathrm{UKT}}=$ universal $\mathrm{k}_{\mathrm{T}}$-points; $\mathrm{p}_{\mathrm{SP}}=$ predicted SmartPulse; $\mathrm{p}_{\mathrm{SP}, \text { ideal }}=$ optimal SmartPulse.

${ }^{a} p_{S P, i d e a l}$ values are in bold in case of misprediction (when different from $p_{S P}$ ).

${ }^{b}$ For each metric and each pulse: Mean (SD) over the testing set. $N_{\text {test }}=53$.

a.

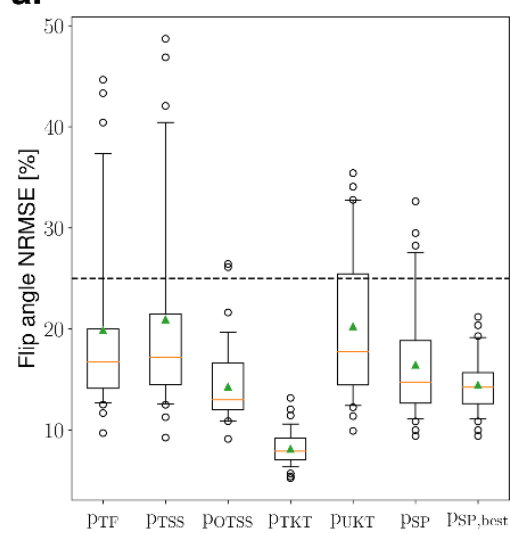

b.

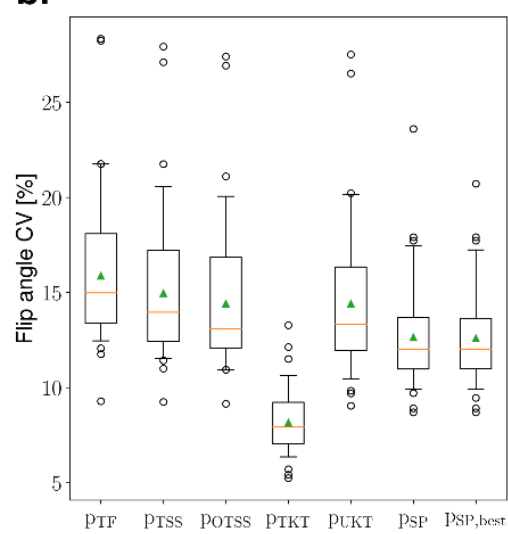

c.

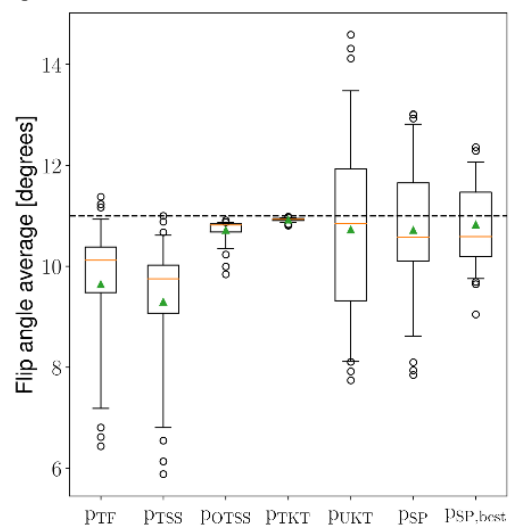

Figure 6 : Distribution of the different metrics used to assess pulse performance: $p_{T F}=$ TrueForm; $p_{\text {TSS }}=$ patient-tailored static RF shimming calculated by the scanner; $p_{\text {OTSS }}=$ optimal patient-tailored static RF shimming; $p_{T K T}=$ tailored $k_{T}$-points; $p_{U K T}=$ universal $k_{T}$-points; $p_{S P}=$ predicted SmartPulse; $p_{S P, \text { ideal }}=$ optimal SmartPulse. Green triangle = mean value; orange line = median; edges of the box = $25^{\text {th }}\left(\right.$ Q1) and $75^{\text {th }}$ (Q3) percentiles; whiskers $=5^{\text {th }}$ and $95^{\text {th }}$ percentiles; circles $=$ outliers. a. Flip angle (FA) NRMSE; the dashed line corresponds to the $25 \%$ threshold. $\boldsymbol{b}$. FA coefficient of variation. $\boldsymbol{c}$. FA average; the dashed line corresponds to the $11^{\circ}$ target. 


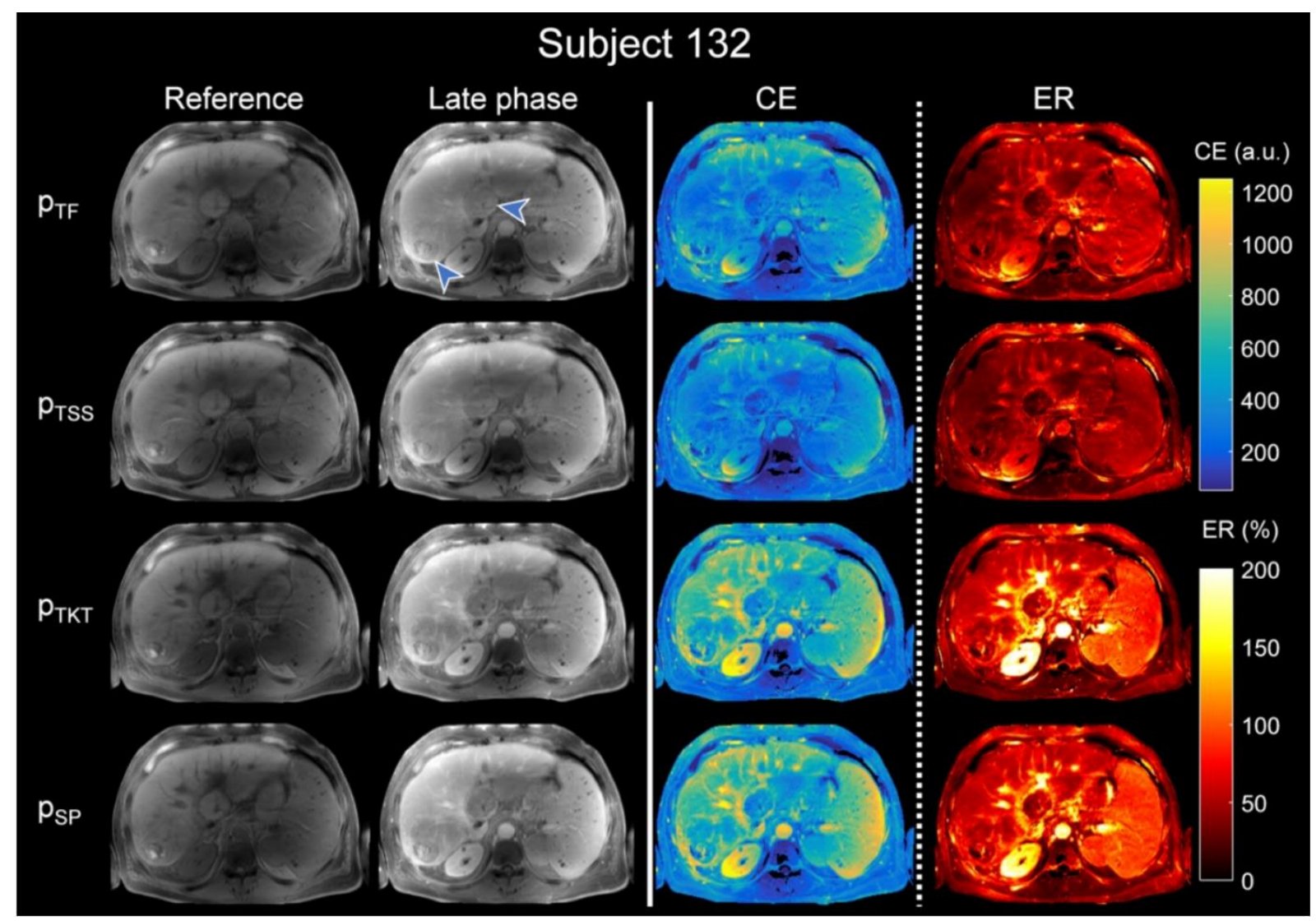

Figure 7 : From left to right: pre-injection (reference), post-injection (late phase), calculated contrast enhancement (CE, Eq. [6]) and enhancement ratio (ER, Eq. [7]) obtained with four pulse techniques applied to a "difficult" subject (TrueForm NRMSE over 25\%, Figure 5). See Table 1 for patient characteristics and Table 2 for performance metrics. $p_{T F}$ : TrueForm; $p_{T S S}$ : patient-tailored static RF shimming; $p_{T K T}$ : patient-tailored $k_{T}$-points; pSP: SmartPulse attributed to the subject. Substantial shading is visible on anatomical, CE and ER images with both $p_{T F}$ and $p_{T S S}$. Two hepatocellular carcinomas (blue arrowheads) are barely visible. $T 1$ contrast is retrieved with both $p_{T K T}$ and $p_{S P}$, and no shading remains in CE nor ER. Additional inhomogeneity can be noticed on anatomical and CE images, due to coil array receiving profile; only ER images are completely free of it.

Figure 7 and Supporting Information Figure S4a focus on two subjects whose TrueForm (ртғ) NRMSE was above $25 \%$. In both cases, using ptr and tailored static RF shimming (ptss) pulses, the enhancement and ER maps lacked intensity and homogeneity. This artifact was largely alleviated with tailored kT-points (рткт) and SmartPulse (psP). For subject 132, $\mathrm{T}_{1}$ contrast was quite poor with $\mathrm{p}$ TF and pTss. 
Some lesions (yellow arrowheads) were harder to see and less delineated than with psp. Scarcely no difference was visible between psp and pTKT images or enhancement maps, despite sensible disparity in NRMSE.

In Figure 8 and Supporting Information Figure S4b, one can compare the different pulses on two "easier" cases: subject 113 (ptF NRMSE of 19\%) and subject 127 (pтF NRMSE of 14\%). Here, all techniques yielded similar results, with no evidence of transmit profile inhomogeneity. Yet, some slight localized enhancement underestimations remained on PTF and pTss acquisitions (arrowheads), but not with pTKT nor psP.

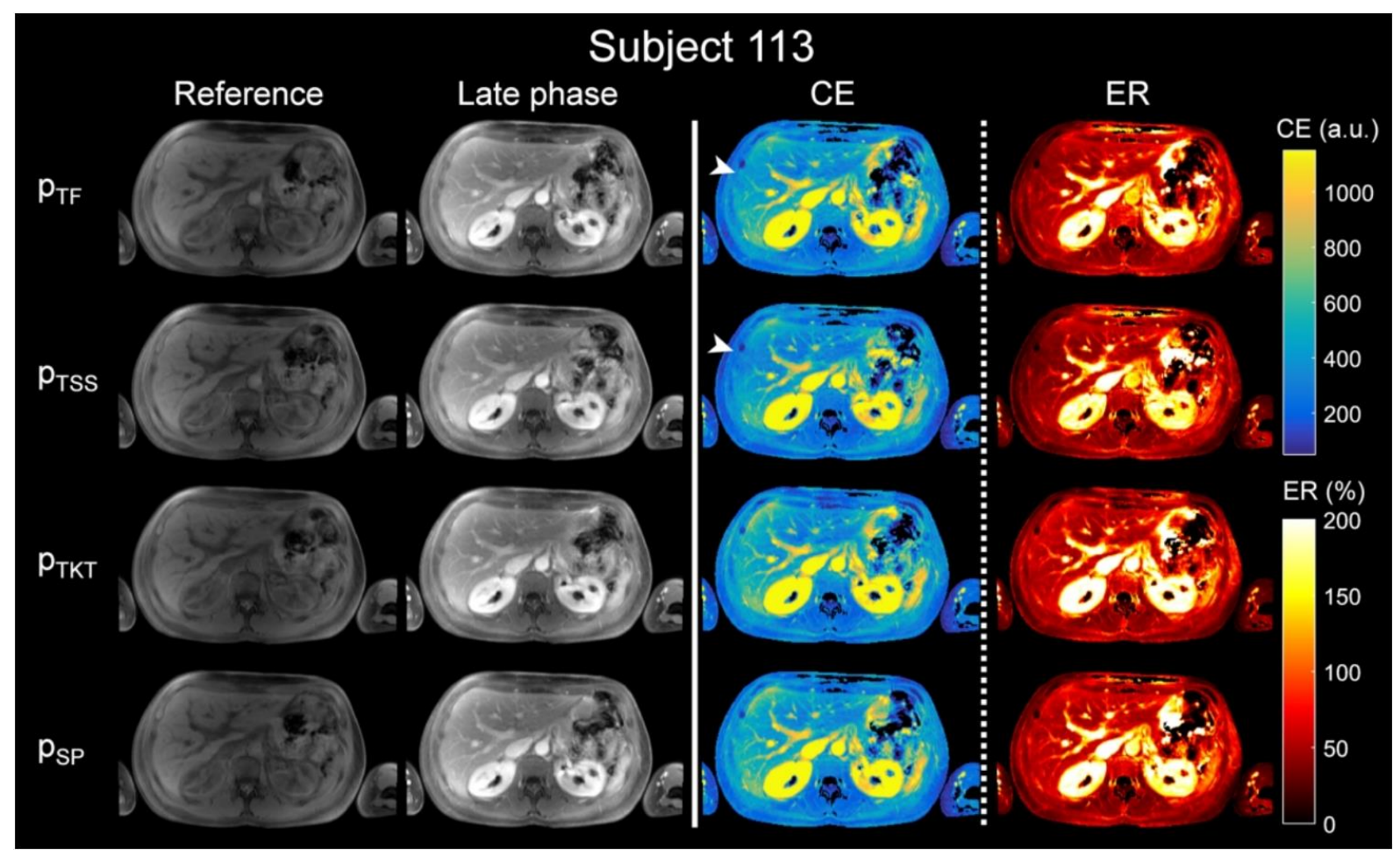

Figure 8 : From left to right: pre-injection (reference), post-injection (late phase), calculated contrast enhancement (CE) and enhancement ratio (ER) obtained with four pulse techniques applied to a "standard" subject (TrueForm NRMSE below 25\%, Figure 5). See Table 1 for patient characteristics and Table 2 for performance metrics. $p_{T F}$ : TrueForm; $p_{T S S}$ : patient-tailored static RF shimming; $p_{T K T}$ : patienttailored kT-points; $p_{S P}$ : SmartPulse attributed to the subject. Arrowhead points out lack of CE in segment 8 (Couinaud's liver segment classification), also noticeable in $E R$ maps, for both $p_{T F}$ and $p_{T S S}$. This artifact is absent from $p_{T K T}$ and $p_{S P}$ maps, which look very much alike. 
Finally, Supporting Information Figure S4c shows an example of SmartPulse misprediction, with subject 115 . The difference between PTF and pSP was clear on ER maps and on the signal level of native images. However, all images showed reasonable inhomogeneity. Indeed, one can see from Table 2 that NRMSE was $14 \%$ with pTF and $23 \%$ with psP, but CV values are the same. The main difference lies in the fact that the mispredicted psP overshot the average FA in subject 115 , while PTF was close to the target. Note however that psp,ideal showed much better behaviour than psp, so that an ideal SmartPulse decision would have solved the problem here.

Bloch-equation simulations of FA maps corresponding to the RF pulses played to acquire the abdominal images shown in Figures 7, 8 and Supporting Information Figure S4 are available in Supporting Information Figure S5.

\section{DISCUSSION AND CONCLUSION}

\subsection{Results Summary}

The SmartPulse process as presented here provided correct pulse assignments in $87 \%$ of the cases. NRMSE simulation results were generally better with psp than either with pTF, pTSS and рuкт. This is even more salient when considering perfect predictions (pSP,ideal). SmartPulse was especially convincing in tempering inhomogeneity in the most difficult subjects. Moreover, these results are obtained almost instantly after completion of the introducing localizer sequence.

Comparing actual acquisition results brought out that SmartPulse does not produce images worse than tailored $\mathrm{k}_{\mathrm{T}}$-points pulse design, despite simulations favourable to the latter. Due to their "universal" nature, SmartPulse kT-points may be more robust to patient motion during the acquisition or between calibration and DCE than their tailored counterpart, not forgetting that $\mathrm{B}_{1}{ }^{+}$maps are acquired in freebreathing, and thus generally do not correspond to breath-hold positions of subjects. Bias in CE and ER was avoided by randomly assigning sequence order for each subject. Additionally, all four acquisitions are performed in the late phase, within a time interval of 4-6 min after injection, in which there should be no significant variation in gadolinium uptake. 
Better results could be obtained by further improvement of the two main aspects of this work: subject classification and pulse performance.

\subsection{Improving Subject Classification}

The difference between training and testing accuracy (85\% in cross-validation, versus $74 \%$ ) indicates model overfitting (48). To reduce this effect, a larger training set would be advisable. Additional or/and different classification tools could also be investigated, but this alone may not solve this issue entirely.

Firstly, the way additional subjects are labelled for training and testing - i.e. selecting the pulse yielding the lowest NRMSE - is different from the way clusters are created. Typical classification errors occur between pulses with similar NRMSE on a given subject, so that a non-optimal choice leading to lower prediction accuracy may not result in significant NRMSE degradation. This is why a tolerance margin of $2 \%$ on NRMSE was used to further analyse classification performance. The fact that 30 of the training subjects were not part of the clustering base and were therefore labelled differently is a particularity of this study, due to the chronology of acquisitions, to the algorithm tuning steps and to the limited size of the database, but is not a requirement of the SmartPulse method itself.

Secondly, there were a few imprecisions in the features used for extraction. Subject's height and weight were provided by the patients themselves, not measured on site, thus leading to slight approximations. Most importantly, abdomen measurements could be much improved by automating localizer placement. This would ensure consistent localizer axial slice locations in the body, and would allow using more than one slice for abdominal dimensions estimation. Another refinement would be to analyse other slice orientations, such as the coronal view - which is already acquired (Figure 4).

\subsection{Improving Pulse Performance}

Flawless class prediction would make NRMSE of all subjects in a $9 \%-21 \%$ range, which should be associated with sufficient excitation uniformity in most - if not all - patients. Still, those results could be improved. In some cases (subjects 96, 103, 114), psp,ideal NRMSE was slightly higher than that of PTF. Also, because of an 
inadequate average FA, some subjects showed relatively high NRMSE despite having low $\mathrm{CV}$.

This issue could be addressed by defining more (finer) clusters. This would require the acquisition of more subjects in order to train the classifier to discriminate between even more categories.

An interesting addition to the current implementation of SmartPulse would be to train a regression model to infer the average FA that the predicted pulse is expected to yield on a given subject, and to adapt the pulse's amplitude accordingly. Preliminary works on this aspect are ongoing and seem promising, but indicate that such a regression model again would require more training data to make precise average FA estimates.

At last, this study did not investigate optimized FA homogenization in the fat, to ensure complete uniform fat suppression, as the default TrueForm excitation mode was chosen for this process. As residual partial fat saturation artifacts were observed in some instances, it is reasonable to believe that homogenizing the $90^{\circ}$ fat FA with dedicated SmartPulses would probably help get rid of those artifacts.

\subsection{Towards a larger database}

For a proof of concept, acquiring $N_{\text {train }}=80$ subjects was deemed sufficient. However, as suggested in the above subsections, building a much larger database would improve A.I. performance. This is not a fundamental issue. It is merely a question of financial means and time impacting acquisition and computing. For computing, the most demanding step is the calculation of $N_{S, 0^{2}}$ NRMSE's required to build the clustering matrix, involving running a Bloch simulator for $N_{S, o}$ tailored pulses applied to $N_{S, 0}$ individuals. Subsequently the agglomerative hierarchical clustering with complete linkage may generate very small clusters as $N_{S, 0}$ increases. In that case, an alternative may be a k-means clustering process.

For classification, more training subjects will translate into a reduction of the model variance, i.e. the test performance will become closer to the training performance. This comes with little cost in extra computing time. 


\subsection{Perspectives}

From the user's point of view, the proposed approach is fast and reliable as it gets rid of all calibrations and optimizations often deemed necessary when using multi-transmit RF coils. This would prove even more useful for ultra-high field body $\mathrm{MRI}$, where the increased number of transmit channels used to perform RF shimming makes calibration and pulse design even more tedious and challenging. Interestingly also, in the opposite situation, SmartPulse could be used on single-channel systems (49), where patient-tailored RF shimming does not exist.

The SmartPulse approach with underlying kT-points pulses is readily implementable for nonselective preparation and 3D imaging to tackle $\mathrm{B}_{1}{ }^{+}$ inhomogeneity in $\mathrm{T}_{1-}, \mathrm{T}_{2}{ }^{*}-, \mathrm{T}_{2}-(24,50)$, or proton-density-weighted imaging. As universal selective pulses, it could also be extended to fast-kz spokes to achieve slab- or slice-selective behaviours and thereby tackle full protocol optimization.

At last, whether SmartPulse could be interesting for organs with more regular geometries such as brains is a relevant question (at UHF). Indeed, provided the classification process can be rendered robust, SmartPulse is always expected to perform better than Universal Pulses as the latter are a particular instance of SmartPulse, with a single cluster. Going to an increasing number of clusters means more and more tailored pulses, therefore better performance. Nevertheless, more investigation would be needed to determine how reliable the classification process could be depending on the organ of interest.

\subsection{Conclusion}

Universal pulse design does not suffice to homogenize excitation in 3T abdomen imaging due to the large variety of abdominal morphology and constitution across the whole population. Once implemented, the proposed method provides a simple and efficient trade-off between tailored and full universal pulse design approaches. Smartpulse performed equally well or better than universal pulses or tailored transmit strategies in most difficult subjects without sacrificing easier ones.

In a dual transmit-channel system, for every single MRI examination, the use of SmartPulses in routine will eventually save between 1 and 2 minutes of $\mathrm{B}_{1}{ }^{+} / \mathrm{B}_{0}$ calibration and tailored RF shimming or pulse design. Even though this gain may 
seem like a small progress with regards to the efforts put into building and exploiting a subject database, proof of concept was brought that A.I. can be used efficiently to yield "off-the-shelf" multiple-transmit RF pulses suitable for everyone. The SmartPulse approach should have a much larger impact at 7T and higher, where 8 to 16 channels are currently proposed by manufacturers.

\section{ACKNOWLEDGEMENTS}

This work is dedicated to the memory of Professor Alain Rahmouni, who passed away on January 26th 2018. Major figure of French radiology, internationally respected, Professor Rahmouni developed an advanced MR teaching and research program at $\mathrm{CHU}$ Henri Mondor imaging department. Bridging early technological innovations in MR to clinical practice was always his ambition. This research study is one of the many projects he supported in this field. Professor Alain Rahmouni will be remembered by all the authors of this manuscript, and many more.

The authors wish to thank all the MRI technicians of Henri Mondor Hospital for their patience and understanding, as well as Lisa Leroi and Gaël Saib for their help in coining a name for the proposed method.

This project was funded by CEA's Programme Transversal, Technologies pour la Santé (Transversal Programme for Health Technologies).

\section{REFERENCES}

1. Willinek WA, Schild HH. Clinical advantages of 3.0 T MRI over 1.5 T. European journal of radiology. 2008;65(1):2-14.

2. Bernstein MA, Huston J, Ward HA. Imaging artifacts at 3.0T. Journal of Magnetic Resonance Imaging. 2006;24(4):735-746.

3. Padormo F, Beqiri A, Hajnal JV, Malik SJ. Parallel transmission for ultrahigh-field imaging. NMR in Biomedicine. 2016;29(9):1145-1161.

4. Kukuk GM, Gieseke J, Nelles M, König R, Andersson M, Muschler E, Mürtz P, Stout J, Nijenhuis M, Träber F, et al. Clinical liver MRI at 3.0 Tesla using parallel RF transmission with patient-adaptive B1 shimming. In: Proc. Intl. Soc. Mag. 
Reson. Med. Vol. 17. 2009. p. 119.

http://cds.ismrm.org/protected/09MProceedings/files/00119.pdf

5. Brink WM, Versluis MJ, Peeters JM, Börnert P, Webb AG. Passive radiofrequency shimming in the thighs at 3 Tesla using high permittivity materials and body coil receive uniformity correction. Magnetic Resonance in Medicine. 2015;76(6):19511956.

6. Franklin K M, Dale B M, Merkle E M. Improvement in B1-inhomogeneity artifacts in the abdomen at $3 T$ MR imaging using a radiofrequency cushion. Journal of Magnetic Resonance Imaging. 2008;27(6):1443-1447.

7. Leroi L, Vignaud A, Sabouroux P, Georget E, Larrat B, Enoch S, Tayeb G, Bonod $\mathrm{N}$, Amadon A, Le Bihan D, et al. B1+ homogenization at 7T using an innovative meta-atom. In: Proc. Intl. Soc. Mag. Reson. Med. Vol. 24. 2016. p. 3531.

8. Nistler J, Renz W. Method for controlling a magnetic resonance system. 2010 Dec 7 [accessed 2016 Nov 4]. http://www.google.ch/patents/US7847554

9. Malik SJ, Keihaninejad S, Hammers A, Hajnal JV. Tailored excitation in 3D with spiral nonselective (SPINS) RF pulses. Magnetic Resonance in Medicine. 2012;67(5):1303-1315.

9. Tomi-Tricot R, Gras V, Mauconduit F, et al. B1 artifact reduction in abdominal DCE-MRI using kT-points: First clinical assessment of dynamic RF shimming at 3T. J Magn Reson Imaging. 2018;47(6):1562-1571. doi:10.1002/jmri.25908

11. Saekho S, Yip C, Noll DC, Boada FE, Stenger VA. Fast-kz three-dimensional tailored radiofrequency pulse for reduced B1 inhomogeneity. Magnetic Resonance in Medicine. 2006;55(4):719-724.

12. Yip C-Y, Fessler JA, Noll DC. Advanced three-dimensional tailored RF pulse for signal recovery in $\mathrm{T}^{*}$-weighted functional magnetic resonance imaging. Magnetic Resonance in Medicine. 2006;56(5):1050-1059.

13. Grissom WA, Khalighi M-M, Sacolick LI, Rutt BK, Vogel MW. Small-tip-angle spokes pulse design using interleaved greedy and local optimization methods. Magnetic resonance in medicine. 2012;68(5):1553-1562.

14. Wu X, Schmitter S, Auerbach EJ, Uğurbil K, Van de Moortele P-F. Mitigating transmit B1 inhomogeneity in the liver at $7 \mathrm{~T}$ using multispoke parallel transmit RF pulse design. Quantitative Imaging in Medicine and Surgery. 2014;4(1):4-10.

15. Cloos MA, Boulant N, Luong M, Ferrand G, Giacomini E, Le Bihan D, Amadon A. kT-points: Short three-dimensional tailored RF pulses for flip-angle 
homogenization over an extended volume. Magnetic Resonance in Medicine. 2012;67(1):72-80.

15. Amadon A, Cloos MA. Method and apparatus for compensating for B1 inhomogeneity in magnetic resonance imaging by nonselective tailored RF pulses. Patent WO2011128847A1, October 2011.

17. Katscher U, Börnert P, Leussler C, van den Brink JS. Transmit SENSE. Magnetic Resonance in Medicine. 2003;49(1):144-150.

18. Zhu Y. Parallel excitation with an array of transmit coils. Magnetic Resonance in Medicine. 2004;51(4):775-784.

19. Geppert C, Nistler J, Renz W, Panagiotelis I, Speckner T. Reduced B1inhomogeneities in breast MRI using optimized RF excitation. In: Proc. Intl. Soc. Mag. Reson. Med. Vol. 16. 2008. p. 2726.

20. Cloos MA, Boulant N, Luong M, Ferrand G, Giacomini E, Hang M-F, Wiggins CJ, Le Bihan D, Amadon A. Parallel-transmission-enabled magnetization-prepared rapid gradient-echo T1-weighted imaging of the human brain at $7 \mathrm{~T}$. Neurolmage. 2012;62(3):2140-2150.

21. Deniz C M, Alon L, Brown R, Zhu Y. Subject- and resource-specific monitoring and proactive management of parallel radiofrequency transmission. Magnetic Resonance in Medicine. 2015;76(1):20-31.

22. Gras V, Vignaud A, Amadon A, Bihan DL, Boulant N. Universal pulses: A new concept for calibration-free parallel transmission. Magnetic Resonance in Medicine. 2017;77(2):635-643.

23. Gras V, Boland M, Vignaud A, Ferrand G, Amadon A, Mauconduit F, Bihan DL, Stöcker T, Boulant N. Homogeneous non-selective and slice-selective paralleltransmit excitations at 7 Tesla with universal pulses: A validation study on two commercial RF coils. PLOS ONE. 2017;12(8):e0183562.

24. Gras V, Mauconduit F, Vignaud A, Amadon A, Le Bihan D, Stöcker T, Boulant N. Design of universal parallel-transmit refocusing $\mathrm{kT}$-point pulses and application to 3D T2 -weighted imaging at 7T. Magnetic Resonance in Medicine. 2018;80(1):5365.

25. Beqiri A, Hoogduin H, Sbrizzi A, Hajnal JV, Malik SJ. Whole-brain 3D FLAIR at 7T using direct signal control. Magnetic Resonance in Medicine. 2018;80(4):15331545. 
26. Mooiweer R, Hajnal JV, Malik SJ. A single-channel universal SPINS pulse for calibration-free homogeneous excitation without PTX. In: Proc. Intl. Soc. Mag. Reson. Med. Vol. 25. 2017. p. 390.

27. Ianni Julianna D., Cao Zhipeng, Grissom William A. Machine learning RF shimming: Prediction by iteratively projected ridge regression. Magnetic Resonance in Medicine. 2018;80(5):1871-1881.

28. Mirfin C, Glover P, Bowtell R. Optimisation of parallel transmission radiofrequency pulses using neural networks. In: Proc. Intl. Soc. Mag. Reson. Med. Vol. 26. Paris, France; 2018. p. 3388.

29. Kierans AS, Leonardou P, Shaikh F, Semelka RC. Body MR imaging: Sequences we use and why. Appl Radiol. 2009;38(5):7-12.

30. Goshima S, Kanematsu M, Kondo H, Shiratori Y, Onozuka M, Moriyama N, Bae KT. Optimal Acquisition Delay for Dynamic Contrast-Enhanced MRI of Hypervascular Hepatocellular Carcinoma. American Journal of Roentgenology. 2009;192(3):686-692.

31. Maniam S, Szklaruk J. Magnetic resonance imaging: Review of imaging techniques and overview of liver imaging. World Journal of Radiology. 2010;2(8):309-322.

32. Gras V, Luong M, Amadon A, Boulant N. Joint design of kT-points trajectories and RF pulses under explicit SAR and power constraints in the large flip angle regime. Journal of Magnetic Resonance. 2015;261:181-189.

33. Tomi-Tricot R, Gras V, Boulant N, Vignaud A, Amadon A. kT-Points Pulse Design at 7T: Optimization of Pulse and Sub-Pulse Durations. In: Magnetic Resonance Materials in Physics, Biology and Medicine. Vol. 29. Vienna, AT; 2016. p. 247400. http://link.springer.com/10.1007/s10334-016-0570-3

34. Setsompop K, Wald LL, Alagappan V, Gagoski BA, Adalsteinsson E. Magnitude least squares optimization for parallel radio frequency excitation design demonstrated at 7 Tesla with eight channels. Magnetic Resonance in Medicine. 2008;59(4):908-915.

35. Hoyos-Idrobo A, Weiss P, Massire A, Amadon A, Boulant N. On variant strategies to solve the magnitude least squares optimization problem in parallel transmission pulse design and under strict SAR and power constraints. IEEE Transactions on Medical Imaging. 2014;33(3):739-748. 
36. Müllner D. fastcluster: Fast Hierarchical, Agglomerative Clustering Routines for R and Python. Journal of Statistical Software. 2013;53(9):1-18.

37. Eichfelder G, Gebhardt M. Local specific absorption rate control for parallel transmission by virtual observation points. Magnetic Resonance in Medicine. 2011;66(5):1468-1476.

38. Blaak E. Gender differences in fat metabolism. Current Opinion in Clinical Nutrition \& Metabolic Care. 2001;4(6):499.

39. Jackson AS, Stanforth PR, Gagnon J, Rankinen T, Leon AS, Rao DC, Skinner JS, Bouchard C, Wilmore JH. The effect of sex, age and race on estimating percentage body fat from body mass index: The Heritage Family Study. International journal of obesity. 2002;26(6):789.

40. The National Electrical Manufacturers Association. Digital Imaging and Communication in Medicine (DICOM). NEMA PS 3 Supplement 23 Structured Reporting. 1997 [accessed 2018 Apr 18]. https://ci.nii.ac.jp/naid/10003989193/

40. Feiweier $T$, Heubes $P$, Speckner T. Method and magnetic resonance system for adjustment of the field strength of RF pulses. Patent US20070145975A1, August 2009.

42. Pedregosa F, Varoquaux G, Gramfort A, Michel V, Thirion B, Grisel O, Blondel M, Prettenhofer $P$, Weiss R, Dubourg V. Scikit-learn: Machine learning in Python. Journal of machine learning research. 2011;12(Oct):2825-2830.

43. Geurts $P$, Ernst D, Wehenkel L. Extremely randomized trees. Machine Learning. 2006;63(1):3-42.

44. Cortes C, Vapnik V. Support-vector networks. Machine Learning. 1995;20(3):273-297.

45. Wu T-F, Lin C-J, Weng RC. Probability estimates for multi-class classification by pairwise coupling. Journal of Machine Learning Research. 2004;5(Aug):975-1005. 46. Jones E, Oliphant T, Peterson P, others. SciPy: Open source scientific tools for Python. 2001.

47. Le Y, Kroeker R, Kipfer HD, Lin C. Development and evaluation of TWIST Dixon for dynamic contrast-enhanced (DCE) MRI with improved acquisition efficiency and fat suppression. Journal of Magnetic Resonance Imaging. 2012;36(2):483491.

48. Domingos $P$. A few useful things to know about machine learning.

Communications of the ACM. 2012;55(10):78. 
49. Eggenschwiler F, O'Brien KR, Gallichan D, Gruetter R, Marques JP. 3D T 2weighted imaging at 7T using dynamic kT-points on single-transmit MRI systems. Magnetic Resonance Materials in Physics, Biology and Medicine. 2016;29(3):347358.

50. Massire A, Vignaud A, Robert B, Le Bihan D, Boulant N, Amadon A. Paralleltransmission-enabled three-dimensional T2-weighted imaging of the human brain at 7 Tesla: 3D T2-Weighted Brain Imaging at 7T. Magnetic Resonance in Medicine. 2015;73(6):2195-2203.

51. Tomi-Tricot R, Gras V, Mauconduit F, Legou F, Boulant N, Gebhardt M, Ritter D, Kiefer B, Zerbib P, Rahmouni A, et al. B1 artifact reduction in abdominal DCE-MRI using kT-points: First clinical assessment of dynamic RF shimming at 3T. Journal of Magnetic Resonance Imaging. 2018;47(6):1562-1571. 


\section{SUPPORTING INFORMATION}

\section{SUPPORTING INFORMATION TABLE S1. Contribution of each feature in the}

classification, as reported by the random forest part of the classification algorithm after training (in decreasing order of importance). Given the dimension of the problem, feature selection should not be a concern. It can be interesting, however, to analyse the importance of features in the classification process. Not surprisingly, reference voltage stands out as the most important feature as it is supposed to summarize the amount of energy required to reach a given level of spin excitation. More surprising, gender turns out to be the next most important feature. Indeed, women tend to have a higher percentage of body fat than men, and fat distribution is sex-dependent (38). Since fat and water have different conductivities and dielectric constants, any information related to fat percentage and location is interesting to estimate RF behavior. Fat generally tends to alleviate $B_{1}$ inhomogeneity contrary to muscle tissue. It was also shown (39) that not only gender but also age have an influence on fat percentage - and its relation to BMI. Apart from the reference voltage and the gender, which lie above the rest, features are quite balanced in terms of information provided. In an additional experiment, a "dummy" feature was added, consisting of a random permutation of the age over all training subjects, so as to evaluate the noise level. This was repeated 20 times (with different permutations): the importance of the lowest-ranking one, age, was 5\% (SD: 0.1\%), compared to $4 \%$ (SD: $1 \%$ ) for the dummy one: all features were above noise level.

\begin{tabular}{lc}
\hline Feature & $\begin{array}{c}\text { Importance } \\
(\%)\end{array}$ \\
\hline Reference voltage & 19 \\
Gender & 14 \\
Weight & 11 \\
Localizer global SAR & 11 \\
Height & 10 \\
Abdominal height & 8 \\
Abdominal width & 8 \\
BMl & 7 \\
Abdominal ratio & 6 \\
Age & 6 \\
\hline
\end{tabular}



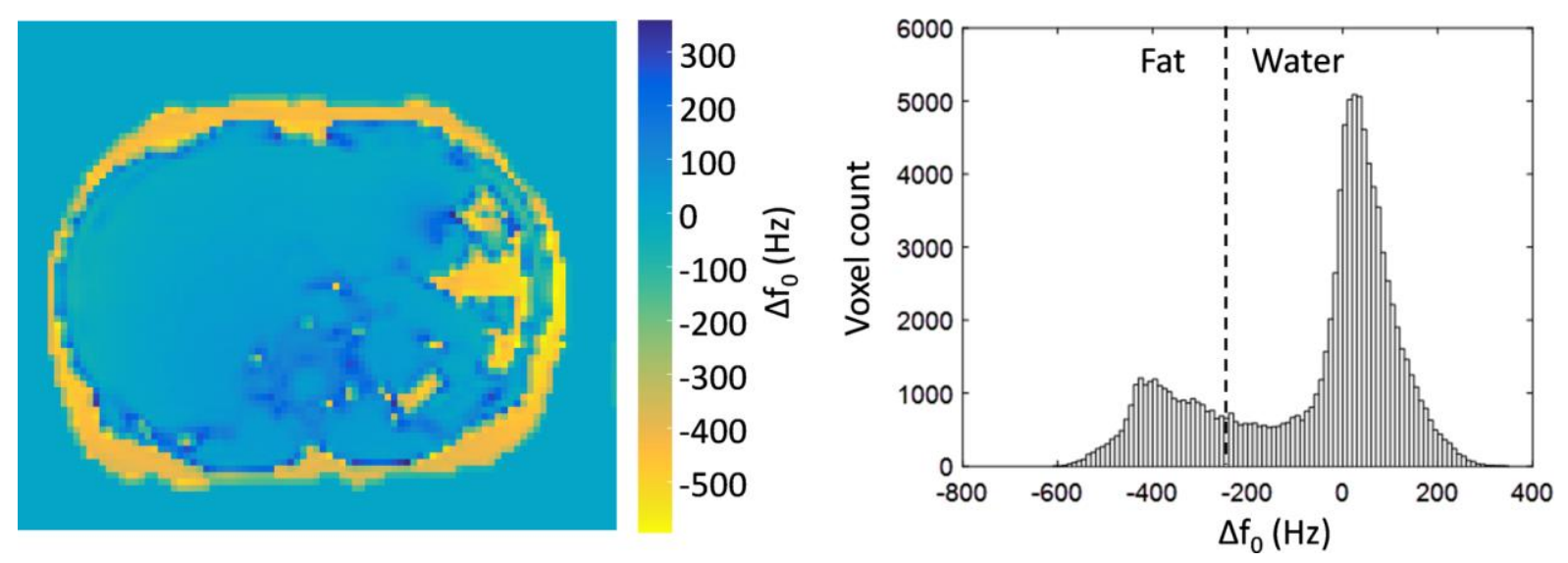

SUPPORTING INFORMATION FIGURE S1. Typical off-resonance map (transverse slice) and associated histogram obtained in the abdomen (mostly liver). Next we describe how these were obtained. The assignment of a voxel to a $\Delta f_{0}$ value was obtained from the phase evolution between two gradient echoes separated by $\triangle T E=$ $0.95 \mathrm{~ms}$. Resonance frequencies could then be distinguished in the [-530 Hz; $+530 \mathrm{~Hz}]$ range without phase wrapping. Now from the raw phase difference, some overlap may occur between the lower-frequency tail of the fat peak and the higher-frequency tail of the water peak. Some basic unwrapping was then performed, exploiting the asymmetrical nature of the histogram, with the water peak centred around $\mathrm{OHz}$ and the fat peak around $-440 \mathrm{~Hz}$. We considered it was very unlikely for a higher-tail water voxel to be misidentified as a lower-tail fat voxel. On the other hand, it was not rare to see the lower tail of fat wrapping up at the other end of the spectrum. We established a threshold, at about $+400 \mathrm{~Hz}$ depending on individuals, above which all voxels were regarded as wrapped-up fat and therefore unwrapped. For each subject, we could then obtain a frequency offset histogram as depicted here. Then to exclude pure fat from the volume of interest retained for pulse design, we set a threshold at $-250 \mathrm{~Hz}$. Obviously, this may not perfectly discriminate fat from water voxels affected by susceptibility-induced frequency offsets, but this threshold was found conservative enough: simulations show that the loss of contrast between fat and water due to poor fat saturation is not rendered worse with $k_{T}$-points excitation than with the TrueForm excitation. 
PROTOCOL START

\section{Breath-hold localizer}

Free-breathing localizer

Calibration ${ }^{\dagger}\left(\Delta \mathrm{f}_{0}, \mathrm{~B}_{1}{ }^{+}\right)$

Several sequences for diagnostics:

- T1w GRE, TRA

- T2w FS/Dixon TSE, TRA

- IVIM DWI EPI, TRA

- T2W TSE/SPACE, COR

- SVS STEAM for fat fraction calculation.

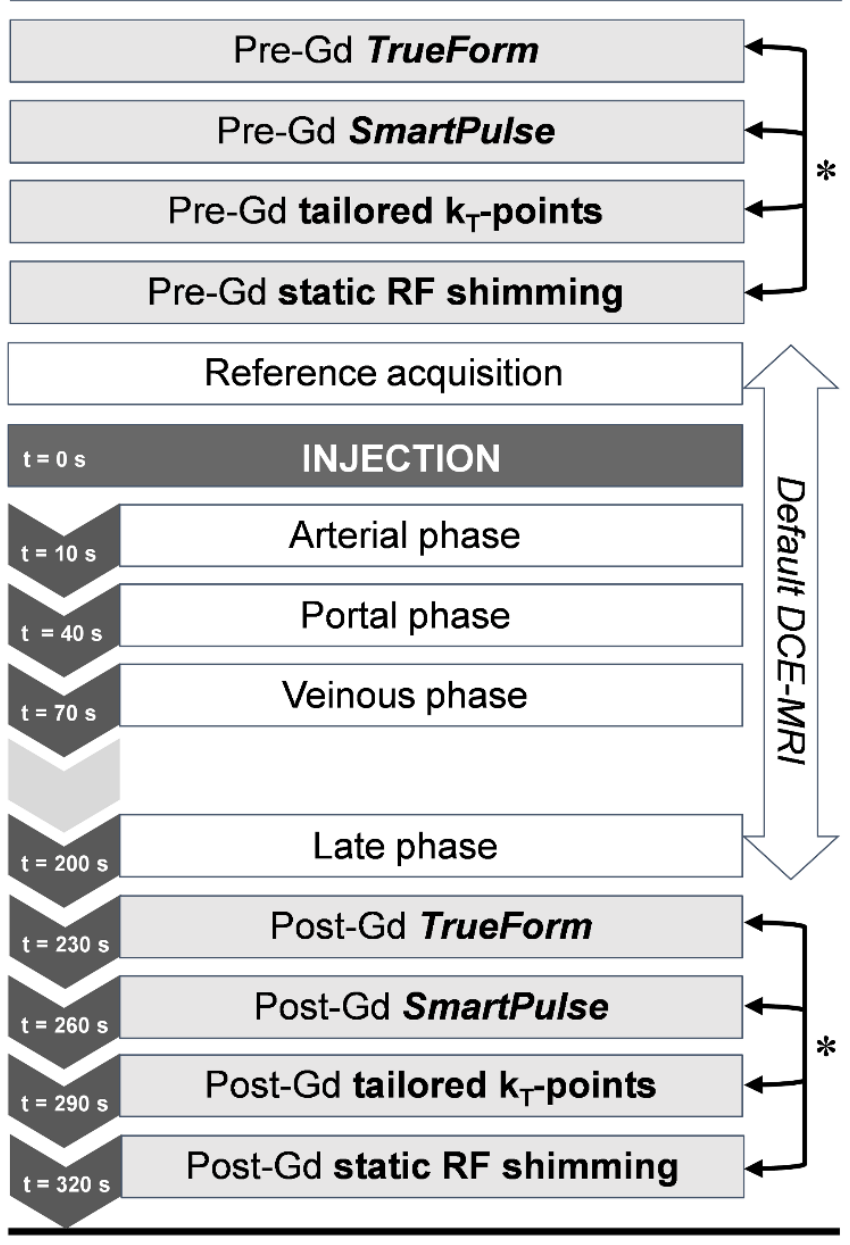

PROTOCOL END

\section{SUPPORTING INFORMATION FIGURE}

S2. Insertion of our sequences (light grey) within the routine liver MRI protocol (white). Injected sequences relevant to this study were acquired at the far end of the acquisition protocol to preserve diagnostics routine timing. Data from the breath-hold localizer was used for subject classification.

* The order of our sequences was randomly assigned.

t The calibration procedure was needed for flip angle simulations and for tailored pulse design, not for SmartPulse nor TrueForm. It included automated manufacturer $\mathrm{B1}^{+}$mapping and a custom-made breath-hold $\Delta f_{0}$ map for proper water fat resolution. Abbreviations: $G R E$ = gradient-recalled echo; TSE = turbo spin-echo; SPACE = sampling perfection with application optimized contrasts using different flip angle evolutions; IVIM = intravoxel incoherent motion; $D W I=$ diffusionweighted imaging; EPI = echo-planar imaging; $\quad$ SVS $=$ single-voxel spectroscopy; STEAM = stimulated echo acquisition mode; DCE-MRI = dynamic contrast-enhanced MRI; TRA = transverse plane; $C O R=$ coronal plane. 


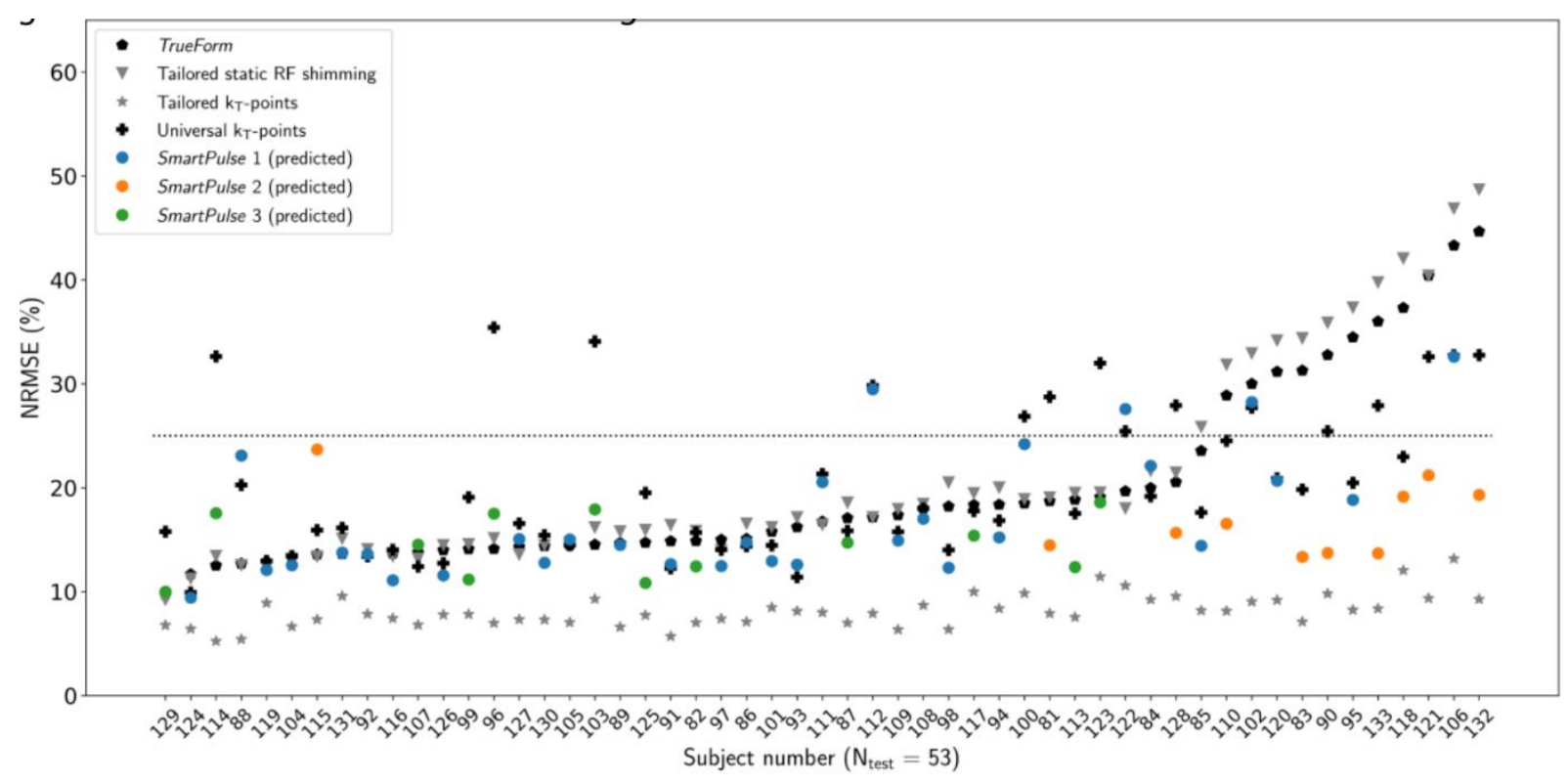

SUPPORTING INFORMATION FIGURE S3. Complementary to Figure 5a: comparison of all simulated pulse designs. Normalized root-mean-square error from the target flip angle (NRMSE) simulation results for all test subjects, sorted in increasing TrueForm inhomogeneity. For each subject, only the predicted SmartPulse is shown. The dashed line indicates 25\% NRMSE. Notice how subjects with a high TrueForm NRMSE are brought towards more acceptable values with SmartPulse. 


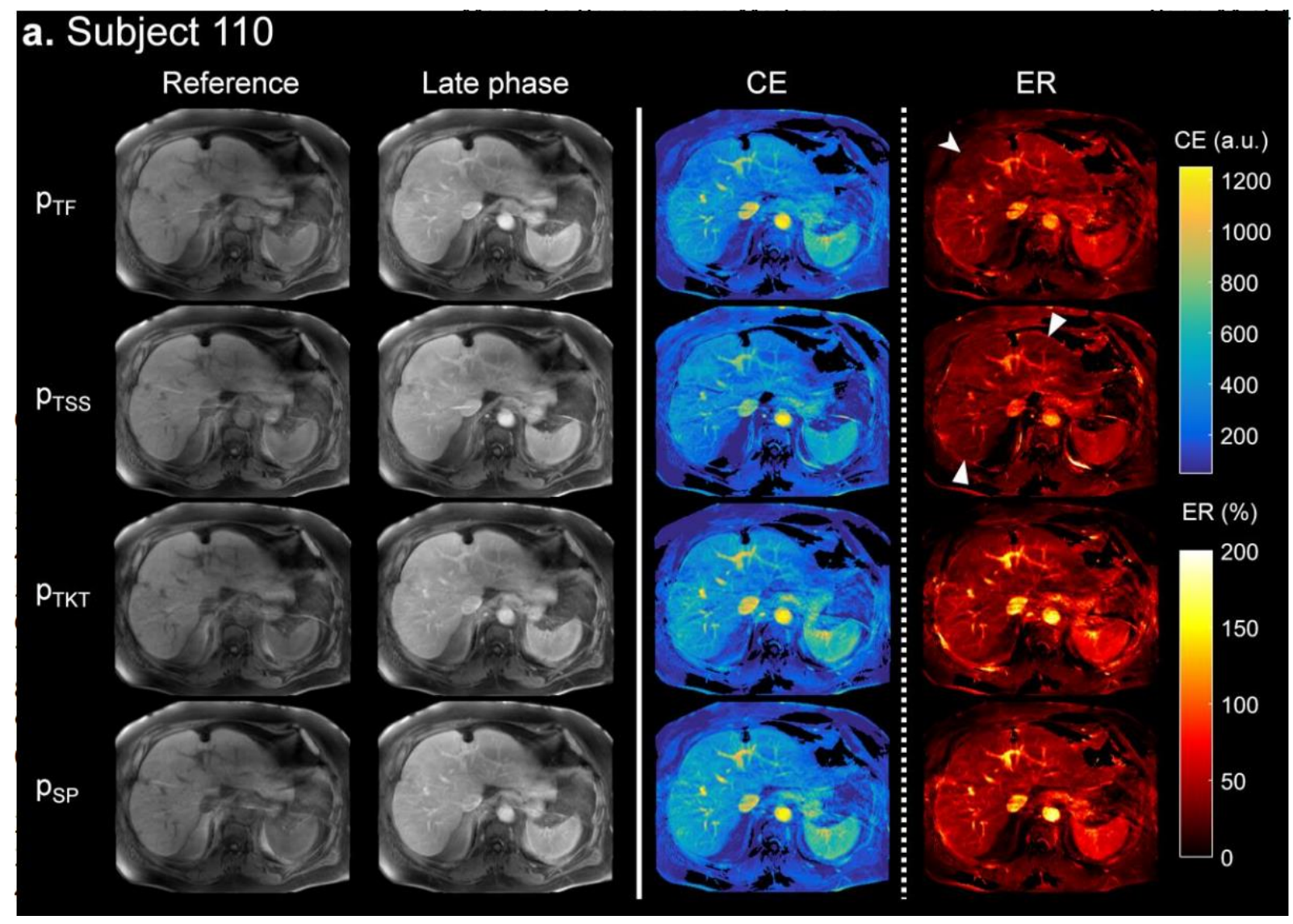

SUPPORTING INFORMATION FIGURE S4. Additional in vivo acquisition results.

From left to right: pre-injection, post-injection, calculated contrast enhancement (CE) and enhancement ratio (ER). $p_{T F}$ : TrueForm; $p_{T S S}$ : patient-tailored static RF shimming; $p_{T K T}$ : patient-tailored $k_{T-p o i n t s ; ~} p_{S P}$ : SmartPulse attributed to the subject. See Table 1 for patient characteristics and Table 2 for performance metrics.

a. "Difficult" subject ( $p_{T F}$ NRMSE over 25\%), complementing Figure 7. Notice the lack of ER in liver segment 5 (Couinaud's liver segment classification) with $p_{T F}$ (notched arrowhead) also visible on the CE image. pTSS results in a more homogeneous $E R$, but at the expense of overall enhancement (full arrowheads). ER is improved with $p_{T K T}$, and even more with $p_{S P}$. 

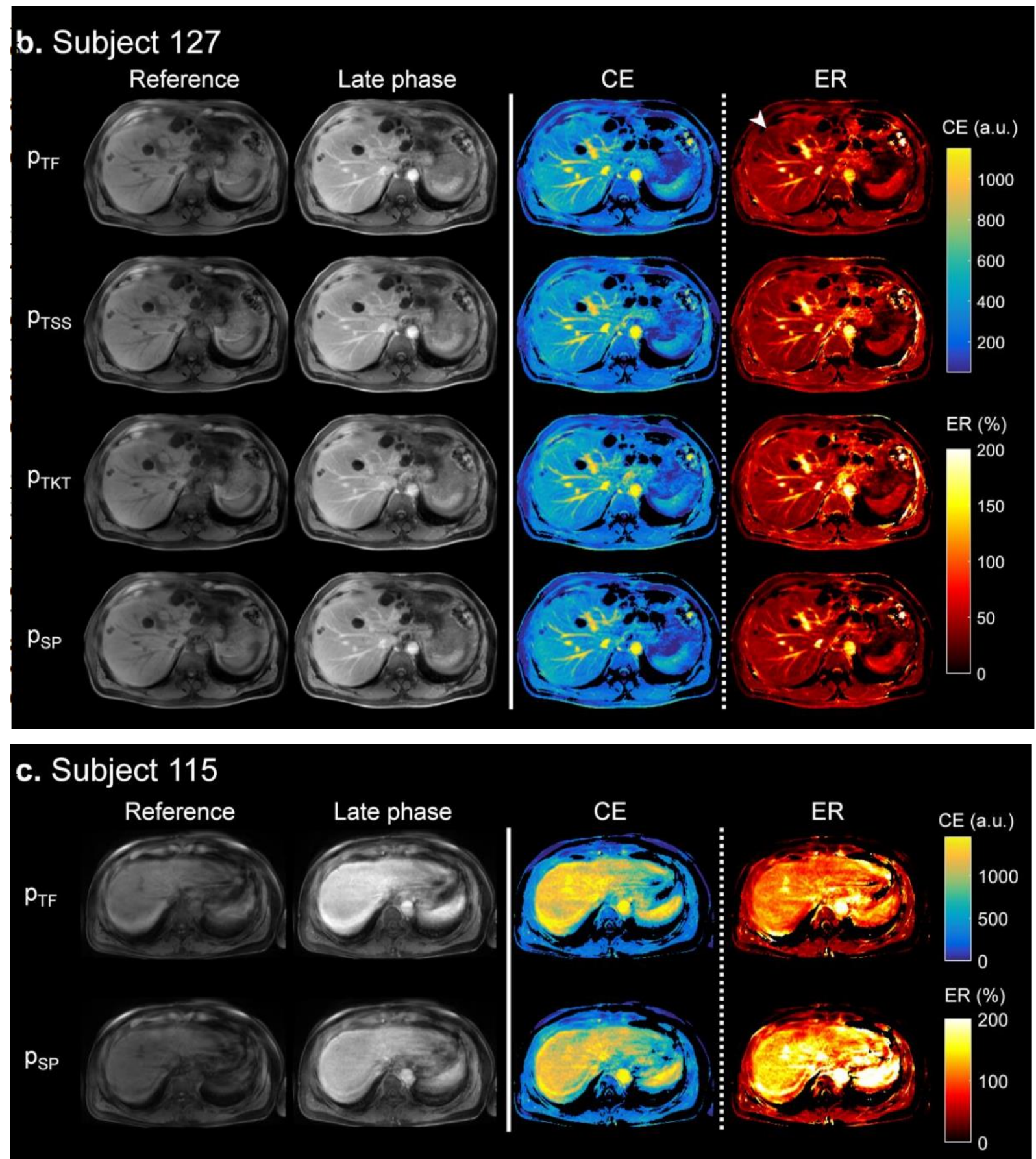

\section{SUPPORTING INFORMATION FIGURE S4 (continued) :}

b. "Standard" subject ( $p_{T F}$ NRMSE below 25\%), complementing Figure 8. All techniques perform similarly, except for a slight CE and ER abnormality (arrowhead) with $p_{T F}$. c. Example of a rare case where predicted $p_{S P}$ was not at all optimal, yielding $23 \%$ NRMSE, compared to $14 \%$ for $p_{T F}$ and $p_{S P \text {,ideal. Homogeneity is the same for both }}$ pulses, but $p_{S P}$ overshoots the flip angle target, hence the overall excessive ER. 


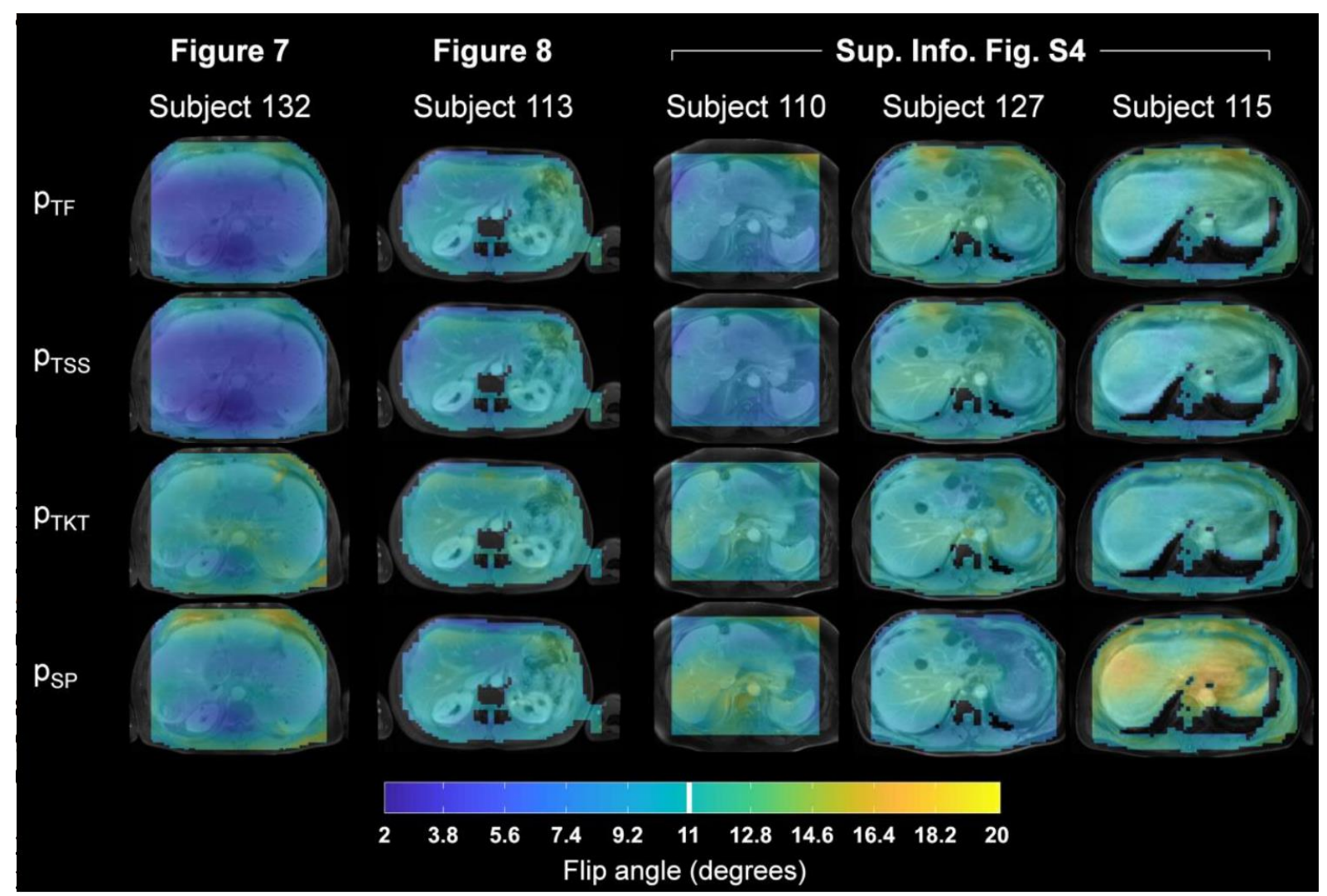

SUPPORTING INFORMATION FIGURE S5. For all subjects shown in the different figures of this work: superposition of acquired post-injection images and simulated flip angle maps obtained with four pulse techniques: TrueForm ( $\left.p_{T F}\right)$, patient-tailored

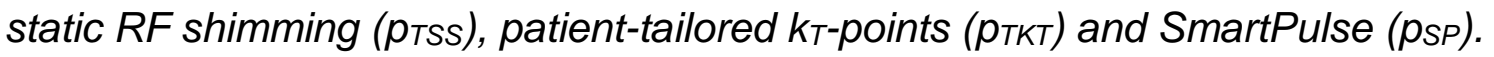
Note that Subject 115 (Sup. Fig. S4) was misclassified by the algorithm: the pulse attributed was not the best possible one, here resulting in a flip angle overshoot. 